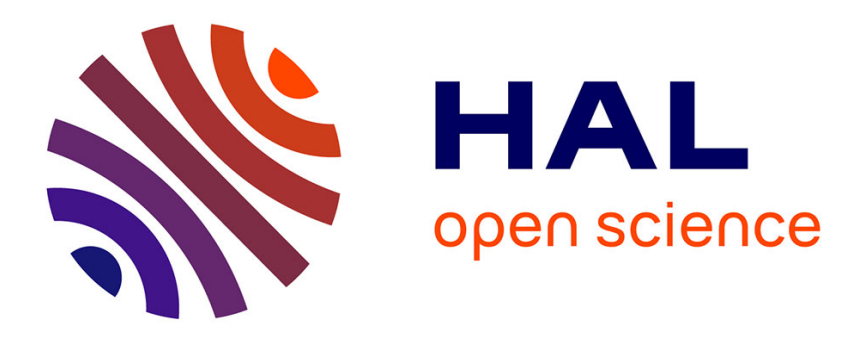

\title{
Intertwining operators for the generalized principal series on a symmetric $R$-space Jean-Louis Clerc
}

\section{To cite this version:}

Jean-Louis Clerc. Intertwining operators for the generalized principal series on a symmetric $R$-space. Transactions of the American Mathematical Society, 2015, 367 (6), pp.4423-4458. 10.1090/S00029947-2014-06327-7 . hal-01277889

\section{HAL Id: hal-01277889 \\ https://hal.science/hal-01277889}

Submitted on 23 Feb 2016

HAL is a multi-disciplinary open access archive for the deposit and dissemination of scientific research documents, whether they are published or not. The documents may come from teaching and research institutions in France or abroad, or from public or private research centers.
L'archive ouverte pluridisciplinaire HAL, est destinée au dépôt et à la diffusion de documents scientifiques de niveau recherche, publiés ou non, émanant des établissements d'enseignement et de recherche français ou étrangers, des laboratoires publics ou privés. 


\title{
Intertwining operators for the generalized principal series on a symmetric $R$-space
}

\author{
Jean-Louis Clerc
}

\begin{abstract}
Three questions about the intertwining operators for the generalized principal series on a symmetric $R$-space are solved : description of the functional kernel, both in the noncompact and in the compact picture, domain of convergence, meromorphic continuation. A large use is made of the theory of positive Jordan triple systems. The meromorphic continuation of the intertwining integral is achieved via a BernsteinSato identity, and a precise description of the poles is obtained.
\end{abstract}

${ }^{0} 2000$ Mathematics Subject Classification : 22E45, 43A80

\section{Introduction}

A symmetric $R$-space is a real (generalized) flag manifold $X=G / P$ ( $G$ a real semi-simple Lie group, $P$ a parabolic subgroup of $G$ ), which is at the same time a Riemannian symmetric space. Or vice-versa, $X$ is a compact Riemannian symmetric space with an "extra" action of a larger (non compact) semisimple Lie group $G$ (the "big group") of diffeomorphisms of $X$ (for this point of view see [21], [29]).

The action of $G$ on $X$ gives rise to a family of representations $\left(\rho_{\lambda}\right)_{\lambda \in \mathbb{C}}$. From a geometric point of view, it is the action of $G$ on the $\lambda$-densities on $X$. From the semisimple harmonic analysis point of view, it is an induced representation from a character (depending on $\lambda$ ) of the parabolic subgroup $P$, usually called the generalized principal series. Associated to this family of representations is a family of intertwining operators $\left(J_{\lambda}\right)_{\lambda \in \mathbb{C}}$ defined $\grave{a}$ la Knapp-Stein, which intertwine $\rho_{\lambda}$ with another representation induced from the opposite parabolic subgroup. The operators correspond to convergent integrals for large values of $\Re \lambda$, and then they are extended meromorphically in the parameter $\lambda$, a special case of a theorem due to Vogan and Wallach (see [30], [31]). The theory however is not explicit enough to give complete information. 
Several authors studied specific examples (the list is not meant to be exhaustive) : rank 1 compact symmetric spaces ([13]), Grassmannian manifolds ([33], [24]), Shilov boundaries of tube-type domains ([26]), spaces associated to real Jordan algebras ([27], [32]), group case([12]), mostly using the fact that $J_{\lambda}$ is a convolution operator on $X$, and computing the spectrum of the operator by use of the harmonic analysis on the compact Riemannian symmetric space $X$. Let also mention the recent work [20] which studies intertwining operators on symmetric R-spaces, which are different from ours.

To my best knowledge, this is the first time where all symmetric $R$-spaces are treated simultaneously and systematically. Following O. Loos (see [16]), symmetric $R$-spaces are approached through the theory of positive Jordan triple systems (PJTS for short).

Positive Hermitian Jordan triple systems (PHJTS for short) and their associated compact Hermitian symmetric spaces play a special (double) role. Viewed with $\mathbb{R}$ as base-field, they are examples of PJTS. On the other hand, a PJTS (resp. a symmetric $R$-space) is a real form of a PHJTS (resp. of a compact Hermitian symmetric space).

The basic strategy is the following : first treat the case of PHJTS/compact Hermitian symmetric spaces with $\mathbb{C}$ as base-field, taking advantage of the holomorphic properties. Then, "restrict" to real forms to obtain the results for PJTS/symmetric $R$-spaces. Restriction should not be understood too strictly. In fact PJTS split in three families : the Hermitian case, the reduced case and the non reduced case, each family demanding its own interpretation of restriction. The Bernstein-Sato identity which is the basic tool for the meromorphic continuation comes in three "cousin" versions, all stemming from the same complex Bernstein-Sato identity.

The example of the spheres, viewed as real projective quadrics may serve as an illustration. The sphere $S^{n}$ is a Riemannian symmetric space of rank 1 , which, when viewed as a real projective quadric, becomes an $R$-space. The one dimensional sphere $S^{1}$ is a real form of the complex projective line $\mathbb{P}^{1}(\mathbb{C})$, a compact Hermitian symmetric space of rank 1 (reduced case). As $S^{2} \simeq$ $\mathbb{P}_{1}(\mathbb{C})$, the two-dimensional sphere $S^{2}$ is a compact Hermitian symmetric space of rank 1 , and it can be realized as a real form of $\mathbb{P}^{1}(\mathbb{C}) \times \overline{\mathbb{P}^{1}(\mathbb{C})}$, where $\overline{\mathbb{P}^{1}(\mathbb{C})}$ is the complex projective line, but with the opposite complex structure (PHJTS case). For $n \geq 3$, the sphere $S^{n}$ is a real form of the complex quadric $\mathbb{Q}_{n}(\mathbb{C})$, which is a compact Hermitian symmetric space of rank 2 (non reduced case).

We finish this introduction by a presentation of the different sections. Section 1 addresses the three main questions precisely :

- explicit form of the kernel of the intertwining operator, both in the 
compact and in the noncompact picture

- domain of convergence

- meromorphic continuation.

In Section 2 the relation between positive Jordan triple systems and symmetric $R$-spaces is exposed with some details, because the literature on the subject is not abundant. Section 3 introduces the complex canonical kernel for a PHJTS, which is used in Section 4 to construct the canonical kernel for a PJTS. Section 5 gives the realization of the canonical kernel in the compact picture, and solves the first question. In section 6, the domain of convergence is determined, thus answering the second question. The rest of the paper (Sections 7-13) is devoted to the third question. In Section 7 , we review the three types of PJTS (PHJTS, reduced, non reduced). In Section 8 we recall the notion of the generic minimal polynomial, which is used in Section 9 to define the fundamental kernel (first its complex version for a PHJTS, then its real version for each of the three types of PJTS) and examine its relation to the canonical kernel. Section 10 is the heart of the paper, as it produces a Bernstein-Sato identity for the special case of the complex fundamental kernel of a PHJTS of tube-type. This is extended in Section 11 to a general PHJTS. In Section 12, a Bernstein-Sato identity is proved for the fundamental kernel of each type of PJTS. Section 13 is devoted to the answer to the third question, namely the meromorphic continuation. A classification of simple PJTS/irreducible symmetric R-spaces is given in an annex. We hope in a future paper to discuss applications to various problems of harmonic analysis over symmetric $R$-spaces (cf [24], [25]).

\section{Symmetric $R$-space, generalized principal series and intertwining operators}

An $R$-space $X$ is a quotient space $X=G / P$, where $G$ is a real semi-simple Lie group (connected and with finite center) and $P$ a parabolic subgroup of $G$. Let $o=e P$ be the origin in $X$. Let $\sigma$ be a Cartan involution of $G$ and let $K=G^{\sigma}$ be the associated maximal compact subgroup of $G$. As $G=K P$, $X$ is homogeneous under the action of $K$. Let $K^{o}$ be the stabilizer of $o$ in $K$, so that $X \simeq K / K^{o}$. The space $X$ is said to be a symmetric $R$-space if there exists an involution $\theta$ of $K$ such that

$$
K_{0}^{\theta} \subset K^{o} \subset K^{\theta}
$$

where $K^{\theta}=\{k \in K, \theta(k)=k\}$ and $K_{0}^{\theta}$ is the connected component in $K^{\theta}$ of the neutral element. 
Let $d \sigma$ be a $K$-invariant measure on $X$ (the normalization will be specified later on). The group $G$ acts smoothly on $X$, and for $g \in G$ and $x \in X$, let $j(g, x)=d \sigma(g x) / d \sigma(x)$ be the Jacobian of $g$ at $x$.

Let $\lambda$ be a complex number. Then $G$ acts naturally on $\lambda$-densities on $X$. It is convenient to trivialize the density bundle by use of the measure $d \sigma$, so that the densities are identified with functions on $X$. For $g$ in $G$ define the operator $\rho_{\lambda}(g)$ on $\mathcal{C}^{\infty}(X)$ by

$$
\rho_{\lambda}(g) f(x)=j\left(g^{-1}, x\right)^{\frac{1}{2}+\lambda} f\left(g^{-1}(x)\right)
$$

$f \in \mathcal{C}^{\infty}(X)$. Then $\rho_{\lambda}$ is a representation of $G$ on $\mathcal{C}^{\infty}(X)$, called the generalized (scalar) principal series of $G$.

The reason for the shift by $\frac{1}{2}$ in the parameter $\lambda$ is to make the following duality property symmetric.

Proposition 1.1. Let $f, \varphi$ be in $\mathcal{C}^{\infty}(X)$

$$
\int_{X}\left(\rho_{\lambda}(g) f\right)(x) \varphi(x) d \sigma(x)=\int_{X} f(y)\left(\rho_{-\lambda}\left(g^{-1}\right) \varphi\right)(y) d \sigma(y) .
$$

The proof is by the change of variable $y=g^{-1}(x)$. The duality relation implies the unitarity of $\rho_{\lambda}$ on $L^{2}(X, d \sigma)$ when $\lambda$ is pure imaginary.

For $\mu$ a complex number, let $\rho_{\mu}^{\sigma}$ be the representation of $G$ defined by $\rho_{\mu}^{\sigma}(g)=\rho_{\mu}(\sigma(g))$. For generic values of $\lambda$, it is known ([14], [30]) that there exists an intertwining operator between $\rho_{\lambda}$ and $\rho_{-\lambda}^{\sigma}$, which is essentially unique. The first goal of the present paper is to give an explicit functional realization of this operator.

Let $\widetilde{c}$ be a continuous everywhere nonnegative function on $X \times X$ satisfying the following covariance property

$$
\widetilde{c}(g(x), \sigma(g)(y))=j(g, x) \widetilde{c}(x, y) j(\sigma(g), y))
$$

for all $x, y \in X \times X$ and $g \in G$. Let $J_{\lambda}$ be the operator (formally) defined by

$$
J_{\lambda}(f)(x)=\int_{X} \widetilde{c}(x, y)^{-\frac{1}{2}+\lambda} f(y) d \sigma(y) .
$$

Proposition 1.2. Let $\lambda \in \mathbb{C}$ such that

$$
\int_{X} \widetilde{c}(o, y)^{-\frac{1}{2}+\Re(\lambda)} d \sigma(y)<+\infty .
$$

Then the operator $J_{\lambda}$ defined by (3) is a continuous operator on $\mathcal{C}^{\infty}(X)$ such that

$$
J_{\lambda} \circ \rho_{\lambda}(g)=\rho_{-\lambda}^{\sigma}(g) \circ J_{\lambda} .
$$


Proof. For $k \in K,(2)$ implies $\widetilde{c}(k(x), k(y))=c(x, y)$ for any $x, y \in X$ and any $k \in K$. Hence $J_{\lambda}$ is a convolution operator on $X$, and the condition (4) guarantees that the operator $J_{\lambda}$ is a convolution operator with an integrable function on $X$, so that $J_{\lambda}$ is a continuous operator on $\mathcal{C}^{\infty}(X)$.

Let $f \in \mathcal{C}^{\infty}(X)$. Then

$$
\begin{gathered}
J_{\lambda}\left(\rho_{\lambda}(g) f\right)(x)=\int_{X} \widetilde{c}(x, y)^{-\frac{1}{2}+\lambda} j\left(g^{-1}, y\right)^{\frac{1}{2}+\lambda} f\left(g^{-1}(y)\right) d \sigma(y) \\
=\int_{X} \widetilde{c}(x, g(z))^{-\frac{1}{2}+\lambda} j(g, z)^{-\frac{1}{2}-\lambda} f(z) j(g, z) d \sigma(z) \\
=j\left(\sigma(g), \sigma\left(g^{-1}\right)(x)\right)^{-\frac{1}{2}+\lambda} \int_{X} \widetilde{c}\left(\sigma\left(g^{-1}\right)(x), z\right)^{-\frac{1}{2}+\lambda} f(z) d \sigma(z) \\
=j\left(\sigma(g)^{-1}, x\right)^{\frac{1}{2}-\lambda} J_{\lambda} f\left(\sigma(g)^{-1}(x)\right)=\left(\rho_{-\lambda}^{\sigma}(g) J_{\lambda} f\right)(x),
\end{gathered}
$$

where we used first the change of variable $y=g(z)$ and next, the covariance property (2) of the kernel $\widetilde{c}$.

Three main questions are addressed in the present paper

- construct, as explicitely as possible a kernel $\widetilde{c}$, and its analogue in the noncompact picture

- determine the domain of convergence of the intertwining integral (4)

- prove the meromorphic continuation in the parameter $\lambda$ to $\mathbb{C}$ and determine the location of the poles.

\section{Symmetric $R$-spaces and positive Jordan triple systems}

The main references for this section are [18] and [19] (see also [10], [11]). For more developments on Jordan triple systems and Jordan pairs, see [17]. For the theory of compact Riemannian symmetric spaces, see [9].

Let $X=G / P$ be a symmetric $R$-space. Keep notation introduced in the beginning of the previous section. The involution $\theta$ can be extended to an involution of $G$ (still denoted by $\theta$ ) and commuting with $\sigma$. Let

$$
\mathfrak{g}=\operatorname{Lie}(G), \quad \mathfrak{p}=\operatorname{Lie}(P), \quad \mathfrak{k}=\operatorname{Lie}(K), \quad \mathfrak{k}^{o}=\operatorname{Lie}\left(K^{o}\right)
$$

Denote by $\sigma$ and $\theta$ the associated involution of the Lie algebra $\mathfrak{g}$.

Let $V=T_{o} X$ be the tangent space of $X$ at $o$. The space $V$ carries two important algebraic structures : a structure of Lie triple system (as does 
the tangent space to any Riemannian symmetric space) and a structure of positive Jordan triple system. Let $\mathfrak{k}=\mathfrak{k}^{o} \oplus \mathfrak{s}$ be the eigenspace decomposition of the restriction of $\theta$ to $\mathfrak{k}$. Regarding $\mathfrak{k}$ as a Lie algebra of vector fields on $X$, the map $\xi \longmapsto \xi(o)$ is an isomorphism of vector spaces from $\mathfrak{s}$ onto $V$. For $v \in V$, let $\widetilde{v}$ be the unique vector field in $\mathfrak{s}$ such that $\widetilde{v}(o)=v$. Then $V$ is turned into a Lie triple system (LTS for short) by

$$
[u, v, w]=[[\widetilde{u}, \widetilde{v}], \widetilde{w}](o) .
$$

Let $P^{-}=\sigma(P)$ be the opposite parabolic subgroup of $P$. Then $H=P^{-} \cap P^{+}$ is a Levi subgroup of $P$. The unipotent radical $N^{+}$(resp. $N^{-}$) of $P$ (resp. of $P^{-}$) is Abelian, and $N^{-}=\sigma\left(N^{+}\right)$. Let $\mathfrak{h}, \mathfrak{n}_{+}, \mathfrak{n}_{-}$be the Lie algebras respectively of $H, N^{+}, N^{-}$. There is a corresponding decomposition of the Lie algebra $\mathfrak{g}$ as

$$
\mathfrak{g}=\mathfrak{n}_{-} \oplus \mathfrak{h} \oplus \mathfrak{n}_{+}=\mathfrak{n}_{-} \oplus \mathfrak{p}
$$

Again regarding $\mathfrak{g}$ as a Lie algebra of vector fields on $X$, the map $\xi \longmapsto \xi(o)$ is an isomorphism of $\mathfrak{n}^{-}$onto $V$. For $v \in V$, denote by $\widehat{v}$ the unique vector field in $\mathfrak{n}^{-}$such that $\widehat{v}(o)=v$. Then $V$ is turned into a positive Jordan triple system (PJTS for short) by letting

$$
\{u, v, w\}=-\frac{1}{2}[[\widehat{u}, \sigma(\widehat{v})], \widehat{w}](o) .
$$

The triple product satisfies the fundamental identities for a Jordan triple system

$$
\begin{gathered}
\{x, y, z\}=\{z, y, x\} \\
\{a, b,\{x, y, z\}\}=\{\{a, b, x\}, y, z\}-\{x,\{b, a, y\}, z\}+\{x, y,\{a, b, z\}\}
\end{gathered}
$$

for all $a, b, x, y, z \in V$. Set

$$
L(x, y) z=\{x, y, z\}, \quad Q(x) y=\{x, y, x\} .
$$

Define the trace form of $V$ by

$$
(x, y)=\operatorname{tr}(L(x, y)) .
$$

The trace form can be verified to be symmetric and positive definite, giving $V$ a structure of positive Jordan triple system. In turn, $V$ becomes equipped with a Euclidean inner product.

An important link between the two trilinear structures on $V$ is the identity

$$
[u, v, w]=2(-\{u, v, w\}+\{v, u, w\})
$$


For a general discussion of the geometric significance of the relation between LTS and JTS structures, see [2].

Let $V$ be a PJTS. A linear operator $g$ on $V$ belongs to the structure $\operatorname{group} \operatorname{Str}(V)$ if, for all $x, y, z$ in $V$,

$$
g\{x, y, z\}=\left\{g x,\left(g^{t}\right)^{-1} y, g z\right\} .
$$

The structure group is a closed (hence Lie) subgroup of $G L(V)$. Its Lie algebra $\mathfrak{s t r}(V)$ is generated by the endomorphisms $L(u, v), u, v \in V$. Moreover, $L(u, v)^{t}=L(v, u)$ for any $u, v \in V$. This implies that $\operatorname{Str}(V)$ is reductive in $G L(V)$. Dilations belong to $\operatorname{Str}(V)$, so that $\mathrm{id}_{V}$ is in $\mathfrak{s t r}(V)$. Let $Z=-\mathrm{id}_{V}$.

A linear operator $k$ on $V$ is an automorphism of $V$ if, for all $x, y, z$ in $V$,

$$
k\{x, y, z\}=\{k x, k y, k z\} .
$$

The automorphisms of $V$ form a Lie group, denoted by $A u t(V)$ and $A u t(V)=$ $\operatorname{Str}(V) \cap O(V)$. The Lie algebra $\mathfrak{a u t}(V)$ of $A u t(V)$ is characterized as $\left\{X \in \mathfrak{s t r}(V), X^{t}=-X\right\}$, and generated by the endomorphisms $(L(u, v)-$ $L(v, u)), u, v \in V$.

Conversely, it is possible to reconstruct the symmetric $R$-space from the PJTS structure on $V$. The process goes first through the Koecher-KantorTits construction.

Proposition 2.1. Let $(V,\{., .,\}$.$) be a PJTS. Let$

$$
\mathfrak{g}=\{(a, T, b), a \in V, T \in \mathfrak{s t r}(V), b \in V\}
$$

and define the bracket of two elements $X=(a, T, b)$ and $X^{\prime}=\left(a^{\prime}, T^{\prime}, b^{\prime}\right)$ by

$$
\left[X, X^{\prime}\right]=\left(T a^{\prime}-T^{\prime} a, 2 L\left(a^{\prime}, b\right)+\left[T, T^{\prime}\right]-2 L\left(a, b^{\prime}\right), T^{\prime t} b-T^{t} b^{\prime}\right) .
$$

i) $\mathfrak{g}$ is a semi-simple Lie algebra.

ii) let

$$
\begin{gathered}
\mathfrak{n}_{-}=\{(a, 0,0), a \in V\} \simeq V \\
\mathfrak{h}=\{(0, T, 0), T \in \mathfrak{s t r}(V)\} \simeq \mathfrak{s t r}(V) \\
\mathfrak{n}_{+}=\{(0,0, b), b\} \simeq V .
\end{gathered}
$$

Then $\mathfrak{g}=\mathfrak{n}^{-} \oplus \mathfrak{h} \oplus \mathfrak{n}^{+}$is a 3-graded Lie algebra, and the gradation is given by $\operatorname{ad} Z$.

iii) the map

$$
\sigma:(a, T, b) \longmapsto\left(b,-T^{t}, a\right)
$$


is a Cartan involution of $\mathfrak{g}$.

iv) the map

$$
\theta:(a, T, b) \longmapsto(-a, T,-b)
$$

is an involution of $\mathfrak{g}$ which commutes with $\sigma$.

$v$ ) identifying $(v, 0,0)$ with $v$ for $v \in V$,

$$
\forall a, b, c \in V, \quad\{a, b, c\}=-\frac{1}{2}[[a, \sigma(b)], c] .
$$

Let

$$
\mathfrak{p}=\mathfrak{h} \oplus \mathfrak{n}^{+}, \mathfrak{k}=\{X \in \mathfrak{g}, \sigma X=X\} .
$$

Then $\mathfrak{k}$ splits under the action of $\theta$ as

$$
\mathfrak{k}=\mathfrak{k}^{o} \oplus \mathfrak{s}
$$

where $\mathfrak{k}^{o} \simeq\left\{T \in \mathfrak{s t r}(V), T^{t}=-T\right\}=\mathfrak{a u t}(V)$ and $\mathfrak{s}=\{(a, 0, a), a \in V\} \simeq V$.

The second step is to integrate these infinitesimal results. Let $G$ be the adjoint group of $\mathfrak{g}$, and denote by $\theta$ and $\sigma$ the involutions of $G$ corresponding to the involutions of $\mathfrak{g}$. Let

- $K=G^{\sigma}$ maximal compact subgroup (hence connected),

- $P$ the normalizer of $\mathfrak{p}$

- $N^{-}=\exp \left(\mathfrak{n}_{-}\right)$Abelian subgroup $\simeq \mathbb{R}^{n}$

- $H=G^{\theta}$ reductive subgroup with Lie algebra $\mathfrak{h}$

- $K^{o}=K \cap P$

Then $K_{0}^{\theta} \subset K^{o} \subset K^{\theta}$. Finally define $X$ as $G / P \simeq K / K^{o}$. Then $X$ is a symmetric $R$-space associated to the PJTS $V$.

Theorem 2.1. The map $V \longmapsto X$ establishes a one-to-one correspondence between (isomorphism classes of) PJTS and symmetric R-spaces.

See [19], [18]. Let us mention that there is also a one-to-one correspondence with the 3-graded real semisimple Lie algebras (see e.g. [3]).

For $v$ in $V$, let $\bar{n}_{v}=\exp (v, 0,0) \in N^{-}$. Then the map

$$
\kappa: V \longrightarrow X, \quad \kappa(v)=\bar{n}_{v}(o)
$$

is a diffeomorphism on an open dense subset of $X$. Hence we can transfer the action of $G$ on $X$ to a (not everywhere defined) action of $G$ on $V$ by

$$
g(x)=\kappa^{-1}(g(\kappa(x))
$$


It turns out to be a rational action. By differentiation, there is a corresponding action of the Lie algebra $\mathfrak{g}$ by differential operators on $V$, explicitly given by

$$
(a, T, b) \longmapsto X(x)=(a+T x+Q(x) b) \frac{\partial}{\partial x} .
$$

By integration, it is possible, to a large extent, to find explicit expressions for the action of elements of $G$ on $V$ (see [18]).

There is a spectral theory for PJTS, which will be used frequently. An element $c$ of $V$ is said to be a tripotent if $\{c, c, c\}=c$. Then $L(c, c)$ is a symmetric operator on $V$, and its eigenvalues belong to $\left\{0, \frac{1}{2}, 1\right\}$, so that, with obvious notation

$$
V=V(c, 1) \oplus V\left(c, \frac{1}{2}\right) \oplus V(c, 0),
$$

the so-called Peirce decomposition of $V$ w.r.t. $c$. When the tripotent $c$ is fixed it will be convenient to set

$$
V_{2}=V(c, 1), \quad V_{1}=V\left(c, \frac{1}{2}\right), \quad V_{0}=V(c, 0) .
$$

The following property will be used frequently : for $i, j, k \in\{0,1,2\}$,

$$
\left\{V_{i}, V_{j}, V_{k}\right\} \subset V_{i-j+k},
$$

where $V_{\ell}$ is assumed to be 0 if $\ell \notin\{0,1,2\}$.

The map $Q(c)$ is 0 on $V\left(c, \frac{1}{2}\right) \oplus V(c, 0)$ and induces an involution on $V(c, 1)$. Hence, the latter decomposes as

$$
V(c, 1)=V^{+}(c, 1) \oplus V^{-}(c, 1) .
$$

Two tripotents $c, d$ are orthogonal if $L(c, d)=0$. If this is the case, then $L(c, c)$ and $L(d, d)$ commute, and $c+d$ is a tripotent.

A subspace $A$ of $V$ is said to be flat if

$$
\{A, A, A\} \subset A, \quad\{x, y, z\}=\{y, x, z\}, \forall x, y, z \in A .
$$

If $\left(c_{1}, c_{2}, \ldots, c_{k}\right)$ is a family of mutually orthogonal tripotents, then $A=$ $\mathbb{R} c_{1} \oplus \mathbb{R} c_{2} \oplus \cdots \oplus \mathbb{R} c_{k}$ is a flat.

There is a (partial) order on the tripotents : $c$ and $d$ being two tripotents, $c$ is smaller than $d$ if $d=c+e$, where $e$ is a tripotent, and $c$ and $e$ are orthogonal. A tripotent which is minimal (0 being removed) for this order is said to be primitive. A tripotent $c$ is primitive if and only if $V^{+}(c, 1)=\mathbb{R} c$. 
A tripotent is said to be maximal if it is maximal for this order. A tripotent $c$ is maximal if and only if $V(c, 0)=\{0\}$.

A Jordan frame is a maximal set of mutually orthogonal primitive tripotents. Let $\left(c_{1}, c_{2}, \ldots, c_{r}\right)$ be a Jordan frame. The operators $L\left(c_{j}, c_{j}\right), 1 \leq$ $j \leq r$ are symmetric and commute to each other, so have a simultaneous decomposition in eigenspaces. More precisely, set

$$
\begin{aligned}
& V_{j j} \quad=\quad V\left(c_{j}, 1\right) \quad j=1, \ldots, r \\
& V_{i j}=V_{j i}=V\left(c_{i}, \frac{1}{2}\right) \cap V\left(c_{j}, \frac{1}{2}\right) \quad 1 \leq i<j \leq r \\
& V_{j 0}=V_{0 j}=V\left(c_{j}, \frac{1}{2}\right) \cap\left(\cap_{k \neq j} V\left(c_{k}, 0\right)\right) \quad j=1, \ldots, r .
\end{aligned}
$$

Then the following Peirce decomposition holds

$$
V=\bigoplus_{0 \leq i \leq j \leq r} V_{i j}
$$

The Peirce decomposition satisfies

$$
\left\{V_{i j}, V_{j k}, V_{k l}\right\} \subset V_{i l} .
$$

and all other brackets are 0 .

Let $c=c_{1}+c_{2}+\cdots+c_{r}$. Then $c$ is a maximal tripotent, and the spectral decomposition of $V$ with respect to $c$ now reads

$$
V(c, 1)=\bigoplus_{1 \leq i \leq j \leq r} V_{i j}, \quad V\left(c, \frac{1}{2}\right)=\bigoplus_{j=1}^{r} V_{o j}, \quad V(c, 0)=\{0\} .
$$

The involution $Q(c)$ induces involutions on each $V_{i j}, 1 \leq i \leq j \leq r$, giving the corresponding decompositions

$$
V_{j j}=\mathbb{R} c_{j} \oplus V_{j j}^{-}, \quad V_{i j}=V_{i j}^{+} \oplus V_{i j}^{-}
$$

If $\left(c_{1}, c_{2}, \ldots, c_{r}\right)$ is a Jordan frame, then the space $\mathbb{R} c_{1} \oplus \mathbb{R} c_{2} \oplus \cdots \oplus \mathbb{R} c_{r}$ is a maximal flat of $V$. Conversely, given a maximal flat subspace $A$ of $V$, there exists a Jordan frame $\left(c_{1}, c_{2}, \ldots, c_{r}\right)$ such that

$$
A=\mathbb{R} c_{1} \oplus \mathbb{R} c_{2} \oplus \cdots \oplus \mathbb{R} c_{r}
$$

Proposition 2.2. Two maximal flat spaces of $V$ are conjugate by some automorphism of $V$. Two Jordan frames of $V$ are conjugate up to order and signs. 
The number of elements in a Jordan frame is called the rank of $V$.

An ideal of $V$ is a subspace $W$ such that $\{W, V, V\} \subset W$ and $\{V, W, V\} \subset$ $W$. The PJTS $V$ is said to be simple if it has no non trivial ideals. A PJTS can be decomposed as a sum of simple ideals in a unique way (up to order).

Proposition 2.3. Let $V$ be a simple PJTS of rank $r$. Let $\left(c_{1}, c_{2}, \ldots, c_{r}\right)$ be a Jordan frame, and let $V=\bigoplus_{0 \leq i \leq j \leq r} V_{i j}$ be the corresponding Peirce decomposition. Then

- for $1 \leq i<j \leq r$, the dimension of $V_{i j}$ (resp. $V_{i j}^{+}, V_{i j}^{-}$) does not depend on $(i, j)$

- for $1 \leq i \leq r$, the dimension of $V_{i o}$ does not depend on $i$

- for $1 \leq i \leq r$, the dimension of $V_{i i}$ does not depend on $i$.

Consequently set

$a=\operatorname{dim} V_{i j}, \quad a_{+}=\operatorname{dim} V_{i j}^{+}, \quad a_{-}=\operatorname{dim} V_{i j}^{-}, \quad b=\operatorname{dim} V_{o i}, \quad c=\operatorname{dim} V_{i i}$.

The numbers $a, b, c$ are called the characteristic numbers of $V$. The genus $p$ of $V$ is defined as

$$
p=(r-1) a+b+2 c .
$$

Observe that $a=0$ if the rank of $V$ is 1 . Otherwise, $a$ is different from 0 . The characteristic number $c$ is always $\geq 1$. If $c=1$ (which amounts to $V(c, 1)=\mathbb{R} c$ for one (equivalently any) primitive tripotent), the PJTS $V$ is said to be reduced. The characteristic number $b$ may be 0 . In this case, $V$ is said to be of tube type or of Jordan algebra type ${ }^{1}$. The reason for the first name is that the dual space to the associated symmetric $R$-space can be realized as a (real) tube-type domain (see [18]). The second name comes from the fact that, for a maximal tripotent $c$, the space $V=V(c, 1)$ can be equipped with a structure of real semi-simple Jordan algebra $V_{c}$ by letting $x_{\cdot c} y=\{x, c, y\}$. The PJTS structure can be recovered from the Jordan algebra structure by the relation $Q(x)=P_{c}(x) \circ Q(c)$, where $P_{c}$ is the usual quadratic operator on the Jordan algebra $V_{c}$. We will come to these points later.

The spectral theory of a PJTS $V$ can be connected to Lie triple system properties of $V \simeq \mathfrak{s}$, mainly through the identity (8).

Let $\left(c_{1}, c_{2}, \ldots, c_{r}\right)$ be a Jordan frame of $V$. Let

$$
A=\left\{x=\sum_{j=1}^{r} t_{j} c_{j}, t_{j} \in \mathbb{R}, 1 \leq j \leq r\right\}
$$

\footnotetext{
${ }^{1}$ For information on Jordan algebras, se [5])
} 
be the corresponding maximal flat subspace of $V$. Then $A$ is a Cartan subspace for the Lie triple structure on $V$, or otherwise said,

$$
\mathfrak{a}=\left\{\widetilde{x}, x=\sum_{j=1}^{r} t_{j} c_{j}, t_{j} \in \mathbb{R}, 1 \leq j \leq r\right\}
$$

is a Cartan subspace of $\mathfrak{s}$. The restricted roots of the pair $(\mathfrak{k}, \mathfrak{a})$ can be described as the linear forms $\lambda \neq 0$ on $\mathfrak{a}$ such that

$$
\mathfrak{s}_{\lambda}=\left\{X \in \mathfrak{s},(\operatorname{ad} H)^{2} X=-\lambda(H)^{2} X, \forall a \in \mathfrak{a}\right\} \neq\{0\} .
$$

Let $x \in V$. Then (see [19] p. 221)

$$
(\operatorname{ad} \widehat{x})^{2} \widehat{y}=(2(-L(x, x)+Q(x)) y)^{\wedge} .
$$

Then, by elementary calculations, for $x=\sum_{j=1}^{r} t_{j} c_{j}$, the operators $L(x, x)$, $Q(x)$ and $(2(-L(x, x)+Q(x)) y)$ can be described by the following matrix notation :

$$
\begin{array}{ccccc} 
& V_{i i}^{-} & V_{i j}^{+} & V_{i j}^{-} & V_{i 0} \\
L(x, x) & t_{i}^{2} & \frac{1}{2}\left(t_{i}^{2}+t_{j}^{2}\right) & \frac{1}{2}\left(t_{i}^{2}+t_{j}^{2}\right) & \frac{1}{2} t_{i}^{2} \\
Q(x) & -t_{i}^{2} & t_{i} t_{j} & -t_{i} t_{j} & 0 \\
2(L(x, x)-Q(x)) & 4 t_{i}^{2} & \left(t_{i}-t_{j}\right)^{2} & \left(t_{i}+t_{j}\right)^{2} & t_{i}^{2}
\end{array}
$$

which gives both the roots and the root spaces. Denote by $\varepsilon_{j}$ the $j$-th coordinate on $\mathfrak{a}$ in the basis $\left(\widetilde{c}_{1}, \ldots, \widetilde{c}_{r}\right)$.

Proposition 2.4. The roots of the pair $(\mathfrak{k}, \mathfrak{a})$ are

- $\pm 2 \varepsilon_{i}, 1 \leq i \leq r$ with multiplicity $c-1$

$\bullet \pm\left(\varepsilon_{i}-\varepsilon_{j}\right), 1 \leq i<j \leq r$ with mutlipliciy $a_{+}$

- $\pm\left(\varepsilon_{i}+\varepsilon_{j}\right), 1 \leq i<j \leq r$ with multiplicity $a_{-}$.

- $\pm \varepsilon_{i}, 1 \leq i \leq r$ with multiplicity $b$.

To this description of Cartan subspaces of $\mathfrak{s}$ corresponds a description of maximal tori of $X$.

Proposition 2.5. Let $\left(c_{1}, c_{2}, \ldots, c_{r}\right)$ be a Jordan frame of $V$ and $A=$ $\sum_{j=1}^{r} \mathbb{R} c_{j}$ the corresponding maximal flat of $V$. Let $\operatorname{Exp}: A \longrightarrow X$ be the map defined by $\operatorname{Exp} v=\exp (\widetilde{v})(o)$.

$i$ ) the image $T=\operatorname{Exp} A$ is a maximal torus of $X$. 
ii) the kernel of Exp is the lattice $\Gamma$ in A given by

$$
\Gamma=\sum_{j=1}^{r} \pi \mathbb{Z} c_{j} .
$$

For the proof see [19] p. 218. This shows that the Riemannian symmetric space $X$ has a cubic unit lattice, and this property is characteristic of the symmetric $R$-spaces among the compact symmetric spaces (see [19]).

As the set of roots has to be a root system $\Sigma$, the determination of the possible roots of the pair $\left(\mathfrak{k}, \mathfrak{k}^{o}\right)$ leaves five possibilities, each giving in turn some information on the values of the possible characteristic numbers.

- type $A_{r-1} \quad \Sigma=\left\{ \pm\left(\varepsilon_{i}-\varepsilon_{j}\right), 1 \leq i \leq j \leq r\right\}$

- type $B_{r} \quad \Sigma=\left\{ \pm \varepsilon_{i}, \pm \varepsilon_{j} \pm \varepsilon_{i}, 1 \leq i<j \leq r\right\}$

- type $C_{r} \quad \Sigma=\left\{ \pm 2 \varepsilon_{i}, \pm \varepsilon_{j} \pm \varepsilon_{i}, 1 \leq i<j \leq r\right\}$

- type $B C_{r} \quad \Sigma=\left\{ \pm \varepsilon_{i}, \pm 2 \varepsilon_{i}, \pm \varepsilon_{i} \pm \varepsilon_{j}, 1 \leq i<j \leq r\right\}$

- type $D_{r} \quad \Sigma=\left\{ \pm \varepsilon_{i} \pm \varepsilon_{j}, 1 \leq i<j \leq r\right\}$

In the vocabulary of PJTS (to be introduced in section 7), they correspond to

- Euclidean type $a_{-}=0, b=0, c=1^{2}$

- reduced, non tube type $b>0, c=1$

- non reduced, tube type $b=0, c>1$

- non reduced, not tube type $b>0, c>1$

- reduced, tube type, and not of Euclidean type $b=0, c=1$.

The Weyl group for $A_{r-1}$ is the permutation group over $\{1,2, \ldots, r\}$. For $B_{r}, B C_{r}$ and $C_{r}$, it is the signed permutation group and for $D_{r}$ it is the signed permutation group with en even number of minus signs.

As a byproduct of this rough classification, observe that for any simple PJTS, $a_{-}=a_{+}$, except for the Euclidean type (for which $a_{-}=0$ ), and (possibly) for the cases of type $D_{2}$, as these are the only cases where the Weyl group does not map $\varepsilon_{1}-\varepsilon_{2}$ to $\varepsilon_{1}+\varepsilon_{2}$. The latter situation occurs only for the PJTS $\mathbb{R}^{p, q}$, for $2 \leq p<q$.

\footnotetext{
${ }^{2}$ The corresponding space $X$ is locally a product of a torus by an irreducible symmetric space, with root system of type $A_{r-1}$. The PJTS of this category correspond to simple Euclidean Jordan algebras and the symmetric spaces are the Shilov boundaries of the irreducible bounded symmetric domains of tube-type.
} 
A consequence of these results is the following integration formula on $X$, in the case where the PJTS $V$ is simple (see [9] Ch. I, theorem 5.10).

Proposition 2.6. For $a=\operatorname{Exp}\left(\sum_{j=1}^{r} t_{j} c_{j}\right)$ in $T$, let

$D(a)=\left|\prod_{i=1}^{r}\left(\sin 2 t_{j}\right)^{c-1} \prod_{1 \leq i<j \leq r} \sin \left(t_{i}-t_{j}\right)^{a_{+}} \prod_{1 \leq i<j \leq r} \sin \left(t_{i}+t_{j}\right)^{a_{-}} \prod_{i=1}^{r}\left(\sin t_{j}\right)^{b}\right|$.

Then, for any continuous function $f$ on $X$ which is invariant by $K^{0}$,

$$
\int_{X} f(x) d \sigma(x)=\int_{T} f(a) D(a) d a .
$$

\section{Compact Hermitian symmetric spaces, positive Hermitian Jordan triple systems, the dual Bergman operator and the complex canonical kernel}

A well-known example of symmetric $R$-spaces is provided by the compact Hermitian symmetric spaces. Their theory is well-known, they are often presented as the dual spaces of the noncompact Hermitian symmetric spaces. We refer to [6]Part III for a presentation of the main results. See also [15], [28].

We slighly modify our notation. The space $\mathbb{X}$ is then a complex manifold which is $\mathbb{G} / \mathbb{P}^{+}$where $\mathbb{G}$ is a complex semi-simple Lie group and $\mathbb{P}^{+}$a certain complex parabolic subgroup. The Cartan involution $\sigma$ is conjugate linear, whereas the involution $\theta$ is complex linear. The maximal compact subgroup of $\mathbb{G}$ is denoted by $U$ and $U^{o}=U \cap \mathbb{P}^{+}$.

This category of spaces corresponds to PJTS admitting a complex structure, that is an operator $J$ on $V$ such that $J^{2}=-$ id and

$$
J\{x, y, z\}=\{J x, y, z\}=-\{x, J y, z\} .
$$

The space $V$ viewed now as a complex vector space is denoted by $\mathbb{V}$ and called a positive Hermitian Jordan triple system (PHJTS for short). In other words, a PHJTS is a complex vector space $\mathbb{V}$ with a map $(x, y, z) \longmapsto$ $\{x, y, z\}$ which is $\mathbb{C}$-linear in $x$ and $z, \mathbb{C}$-conjugate linear in $y$ and satisfies the algebraic conditions (6) and (7) plus a positivity condition. The trace form of $\mathbb{V}$ given by

$$
(x, y)=\operatorname{tr}_{\mathbb{C}}(L(x, y))
$$


has to be a positive-definite Hermitian form. If $L$ is a $\mathbb{C}$-linear endomorphism of $V$, denote by $L^{*}$ its adjoint with respect to the trace form. In particular, for any $x, y \in V, L(x, y)^{*}=L(y, x)$.

Let $\left(c_{1}, c_{2}, \ldots, c_{r}\right)$ be a Jordan frame of a simple PHJTS $V$. The eigenspaces entering in the corresponding Peirce decomposition are now complex vector subspaces. In particular, $V_{j j}=\mathbb{C} c_{j}, 1 \leq j \leq r$ (hence $c=2$ ) and we let $a_{\mathbb{C}}=\operatorname{dim}_{\mathbb{C}} V_{i j}, b_{\mathbb{C}}=\operatorname{dim}_{\mathbb{C}} V_{j 0}$ for $1 \leq i, j \leq r$, so that $a=2 a_{\mathbb{C}}, b=2 b_{\mathbb{C}}$. The complex genus is defined as $p_{\mathbb{C}}=(r-1) a_{\mathbb{C}}+b_{\mathbb{C}}+2$, so that $p=2 p_{\mathbb{C}}($ cf $(9))$.

The group $\operatorname{Str}(\mathbb{V})$ is defined along the same line as before and is now a complex Lie subgroup of $G L(\mathbb{V})$. The group of automorphisms $\operatorname{Aut}(\mathbb{V})$ is equal to $\operatorname{Str}(\mathbb{V}) \cap U(\mathbb{V})$.

The action of $\mathbb{G}$ on $\mathbb{X}$ can be transfered to a rational holomorphic action on $\mathbb{V}$ as before. For $g \in \mathbb{G}$ defined at $x \in \mathbb{V}$, we let $J(g, x)$ be the differential of $g$ at $x$. The differential $J(g, x)$ can be shown to belong to $\operatorname{Str}(\mathbb{V})$.

Let $\tau=\theta \circ \sigma$. The fixed points set of this third involution of $\mathbb{G}$ is the group of holomorphic isometries of the noncompact Riemannian symmetric space dual to $\mathbb{X}$. The Bergman operator is defined for $x, y \in \mathbb{V}$ by

$$
B(x, y)=\mathrm{id}_{V}-2 L(x, y)+Q(x) Q(y)
$$

This is a $\mathbb{C}$-linear endomorphism of $\mathbb{V}$, and, as a function of $x$ (resp. $y$ ) it is holomorphic (resp. conjugate holomorphic). The following proposition gives its most important property (see [28] (ch II, Lemma 5.2). .

Proposition 3.1. For $g \in \mathbb{G}$ and $x, y \in \mathbb{V}$ such that $g(x)$ and $\tau(g)(y)$ are defined,

$$
B(g(x), \tau(g)(y))=J(g, x) B(x, y) J(\tau(g), y)^{*} .
$$

This formula is the holomorphic extension to $\mathbb{G}$ of the covariance property of the action of the isometry group of the bounded symmetric domain which is the dual Riemannian symmetric space of $\mathbb{X}$. We need a version of this property for $\mathbb{X}$.

Let

$$
C(x, y)=B(x,-y)=\operatorname{id}_{V}+2 L(x, y)+Q(x) Q(y)
$$

which we call the dual Bergman operator of $\mathbb{V}$.

Proposition 3.2. For $g \in \mathbb{G}$ and $x, y \in \mathbb{V}$ such that $g(x)$ and $\sigma(g)(y)$ are defined,

$$
C(g(x), \sigma(g)(y))=J(g, x) C(x, y) J(\sigma(g), y)^{*} .
$$


Proof. Let $\iota=\exp (i \pi Z)$. Then $\operatorname{Ad} \iota=\theta$, so that the involution $\theta$ of $\mathbb{G}$ coincides with the inner automorphism $g \longmapsto \iota \circ g \circ \iota$. Moreover, for $x \in \mathbb{V}$, $\iota(x)=-x$. Hence, (12) can be rewritten as

$$
B(g(x),-\sigma(g)(-y))=J(g, x) B(x, y) J(\iota \circ \sigma(g) \circ \iota, y)^{*} .
$$

Now, by the chain rule,

$$
J(\iota \circ \sigma(g) \circ \iota, y)=-\operatorname{id}_{V} \circ J(\sigma(g),-y) \circ\left(-\operatorname{id}_{V}\right)=J(\sigma(g),-y) .
$$

Now change $y$ to $-y$ to obtain (14).

The complex canonical kernel on $\mathbb{V} \times \mathbb{V}$ is defined by

$$
c_{\mathbb{C}}(x, y)=\operatorname{det}_{\mathbb{C}} C(x, y) .
$$

Proposition 3.3. Let $\mathbb{V}$ be a PHJTS, and let $c_{\mathbb{C}}(x, y)$ be its complex canonical kernel.

i) $c_{\mathbb{C}}$ is holomorphic in $x$ and antiholomorphic in $y$.

ii) For any $x, y \in \mathbb{V}$ and $g \in \mathbb{G}$ such that $g(x)$ and $\sigma(g)(y)$ are defined

$$
c_{\mathbb{C}}(g(x), \sigma(g) y)=j(g, x) c_{\mathbb{C}}(x, y) \overline{j(\sigma(g), y)},
$$

where $j(g, x)$ is the (complex) Jacobian of $g$ at $x$.

iii) Assume $V$ is simple and let $\left(c_{1}, c_{2}, \ldots, c_{j}\right)$ be a Jordan frame in $\mathbb{V}$, and let $x=\sum_{j=1}^{r} x_{j} c_{j}$, where $x_{j} \in \mathbb{C}, 1 \leq j \leq r$. Then

$$
c_{\mathbb{C}}(x, x)=\prod_{j=1}^{r}\left(1+x_{j} \overline{x_{j}}\right)^{p_{\mathbb{C}}} .
$$

The points $i$ ) and $i i$ ) are easy consequences of the definition of $c_{\mathbb{C}}$, and iii) is proved using (10).

\section{The dual Bergman operator and the canonical kernel for a PJTS}

Let $\mathbb{V}$ be a PHJTS. An involution $\alpha$ of $\mathbb{V}$ is, by definition, a conjugate linear map of $\mathbb{V}$ which is involutive $(\alpha \circ \alpha=\mathrm{id})$ and satisfies

$$
\{\alpha x, \alpha y, \alpha z\}=\alpha\{x, y, z\} .
$$

Then $V=\{x \in \mathbb{V}, \alpha(x)=x\}$ with the induced triple product is a PJTS. The PJTS $V$ is said to be a real form of the PHJTS $\mathbb{V}$. Now conversely, 
any PJTS is, in a canonical way, a real form of a PHJTS. In fact, let $V$ be a PJTS. Then let $\mathbb{V}=V \otimes_{\mathbb{R}} \mathbb{C}$ be its complexification and extend the Jordan product $\{x, y, z\}$ to $\mathbb{V}$ in a $\mathbb{C}$-linear way in $x$ and $z$ and in a conjugate linear way in $y$. Then $\mathbb{V}$ is a PHJTS, and the conjugation with respect to $V$ is an involution of $\mathbb{V}$. The space $\mathbb{V}$ is called the Hermitification of $V$.

Let $V$ be a PJTS, let $\mathbb{V}$ be its Hermitification, and let ${ }^{-}: z \mapsto \bar{z}$ be the conjugation of $\mathbb{V}$ w.r.t. $V$. There corresponds an involution of $\mathbb{G}$, given by $g \longmapsto{ }^{-} \circ g \circ^{-}$and the fixed points set $G$ of this involution is a real form of $\mathbb{G}$. It is a real semisimple Lie group. Let $P=\mathbb{P}^{+} \cap G$ which is a parabolic subgroup of $G$. Then the quotient space $X=G / P$ is a real form of $\mathbb{X}=\mathbb{G} / \mathbb{P}^{+}$. The Cartan involution $\sigma$ of $\mathbb{G}$ restricts to a Cartan involution of $G$, and $X=(G \cap U) /\left(G \cap U^{o}\right)=K / K^{o}$ is realized as a compact Riemannian symmetric space.

As for the case of PHJTS, define the dual Bergman operator of $V$ as

$$
C(x, y)=\mathrm{id}+2 L(x, y)+Q(x) Q(y) .
$$

Then (14) implies, for $x, y \in V$ and $g \in G$ such that $g$ is defined at $x$ and $\sigma(g)$ defined at $y$,

$$
C(g(x), \sigma(g)(y))=J(g, x) C(x, y) J(\sigma(g), y)^{t} .
$$

Define the canonical kernel on $V \times V$ to be

$$
c(x, y)=|\operatorname{Det} C(x, y)| .
$$

For $g \in G$ and $x \in V$ and $g$ defined at $x$, let $j(g, x)=\mid$ Det $J(g, x) \mid$ be the Jacobian of $x$ at $g$.

Proposition 4.1. Let $g \in G$, and $x, y \in V$ such that $g$ is defined at $x$ and $\sigma(g)$ is defined at $y$. Then

$$
c(g(x), \sigma(g)(y))=j(g, x) c(x, y) j(\sigma(g), y) .
$$

This property follows from the covariance property of the dual Bergman operator.

Proposition 4.2. Let $V$ be a simple PJTS of rank $r$ and let $\left(c_{1}, c_{2}, \ldots, c_{r}\right)$ be a Jordan frame of $V$. Let $x=\sum_{j=1}^{r} x_{j} c_{j}$, and set $x_{0}=0$.

i) for $0 \leq i \leq j \leq r$ and $y_{i j} \in V_{i j}$

$$
C(x, x) y_{i j}=\left(1+x_{i}^{2}\right)\left(1+x_{j}^{2}\right) y_{i j} .
$$


ii)

$$
c(x, x)=\left(\prod_{j=1}^{r}\left(1+x_{j}^{2}\right)\right)^{p}
$$

where $p$ is the genus of $V$ (cf (9)).

Proof. The first statement is a consequence of the following identities, for $0 \leq i \leq j \leq r$

$$
\begin{gathered}
C(x, x)=\mathrm{id}+2 L(x, x)+Q(x)^{2} \\
L(x, x)=\sum_{j=1}^{r} x_{j}^{2} L\left(c_{j}, c_{j}\right) \\
Q(x) y_{i j}=x_{i} x_{j} Q(c) y_{i j}
\end{gathered}
$$

where $c=\sum_{j=1}^{r} c_{j}$, and the fact that $Q(c)^{2}$ is the projection on $V(c, 1)$. As $C(x, x)$ is scalar on each $V_{i j}$, its determinant is easily computed.

The canonical kernel satisfies the symmetry property

$$
c(x, y)=c(y, x)
$$

for all $x, y \in V$, which is a consequence of the symmetry property of the dual Bergman operator

$$
C(y, x)=C(x, y)^{t} .
$$

The next statement will play an important role in extending results from the PJTS of Jordan algebra type to general PJTS.

Proposition 4.3. Let $c$ be a maximal tripotent in $V$, and let $V=V_{2} \oplus V_{1}$ be the associated Peirce decomposition. Then the following identity holds:

$$
c\left(x_{2}+x_{1}, y_{2}\right)=c\left(x_{2}, y_{2}\right) .
$$

for any $x_{1} \in V_{1}, x_{2} \in V_{2}$ and $y_{2} \in V_{2}$.

Proof. The linear operators on $V$ will be denoted in matrix form with respect to the decomposition $V=V_{2}+V_{1}$. Let $x=x_{2}+x_{1}$ be an arbitrary element of $V$. Then $L\left(x, y_{2}\right)$ has the following form :

$$
L\left(x, y_{2}\right)=\left(\begin{array}{cc}
\left\{x_{2}, y_{2}, .\right\} & 0 \\
\left\{x_{1}, y_{2}, .\right\} & \left\{x_{2}, y_{2}, .\right\}
\end{array}\right) .
$$


Similarly,

$$
Q\left(y_{2}\right)=\left(\begin{array}{cc}
\left\{y_{2}, ., y_{2}\right\} & 0 \\
0 & 0
\end{array}\right)
$$

and

$$
Q(x)=\left(\begin{array}{cc}
\left\{x_{2}, ., x_{2}\right\} & 2\left\{x_{1}, ., x_{2}\right\} \\
2\left\{x_{1}, ., x_{2}\right\} & \left\{x_{1}, ., x_{1}\right\}
\end{array}\right)
$$

so that

$$
C\left(x, y_{2}\right)=\left(\begin{array}{cc}
1_{W_{2}}+2 L\left(x_{2}, y_{2}\right)_{\mid V_{2}}+Q\left(x_{2}\right)_{\mid V_{2}} Q\left(y_{2}\right)_{\mid V_{2}} & 0 \\
& \\
\star & 1_{W_{1}}+2\left\{x_{2}, y_{2}, .\right\}
\end{array}\right) .
$$

Hence,

$$
c\left(x, y_{2}\right)=c\left(x_{2}, y_{2}\right)
$$

\section{The canonical kernel in the compact picture}

We use freely of the notation introduced so far. Let $V$ be a simple PJTS. In this section, we transfer the previous results in the compact picture, using the map $\kappa$. Some preliminary results will be needed.

Proposition 5.1. The measure on $V$ define by

$$
f \longmapsto \int_{V} f(x) c(x, x)^{-\frac{1}{2}} d x
$$

is invariant under the action of $K$.

Proof. Let $k$ be in $K$. Then $\sigma(k)=k$, so that, at any point $x$ where $k(x)$ is defined,

$$
c(k(x), k(x))=j(k, x)^{2} c(x, x) .
$$

Now

$$
\begin{gathered}
\int_{V} f\left(k^{-1}(x)\right) c(x, x)^{-\frac{1}{2}} d x=\int_{V} f(y) c(k(y), k(y))^{-\frac{1}{2}} j(k, y) d y \\
=\int_{V} f(y) c(y, y)^{-\frac{1}{2}} d y
\end{gathered}
$$


This result can be used to normalize the $K$-invariant measure $d \sigma$ on $X$, by the condition that

$$
\int_{X} f(x) d \sigma=\int_{V} f(\kappa(v)) c(v, v)^{-\frac{1}{2}} d v .
$$

In turn, it implies, that for this normalization of the measure $d \sigma$

$$
j(\kappa, v)=\frac{d \sigma(\kappa(v))}{d v}=c(v, v)^{-\frac{1}{2}} .
$$

Proposition 5.2. Let $\left(c_{1}, c_{2}, \ldots, c_{r}\right)$ be a Jordan frame of $V$. For $\boldsymbol{\theta}=$ $\left(\theta_{1}, \theta_{2}, \ldots, \theta_{r}\right) \in(\mathbb{R} / \pi \mathbb{Z})^{r}$,

$$
\kappa\left(\sum_{j=1}^{r} \tan \theta_{j} c_{j}\right)=\operatorname{Exp}\left(\sum_{j=1}^{r} \theta_{j} c_{j}\right) .
$$

For a proof, see [19]. In the sequel, we let $a_{\boldsymbol{\theta}}:=\operatorname{Exp}\left(\sum_{j=1}^{r} \theta_{j} c_{j}\right)$.

The following theorem answers the first question we addressed at the end of section 1 .

Theorem 5.1. There exists a unique everywhere nonnegative continuous function $\widetilde{c}$ on $X \times X$ such that

i) $\widetilde{c}\left(o, a_{\boldsymbol{\theta}}\right)=\left(\prod_{j=1}^{r} \cos ^{2} \theta_{j}\right)^{\frac{p}{2}}$, for all $\boldsymbol{\theta} \in(\mathbb{R} / \pi \mathbb{Z})^{r}$.

ii) for all $s, t \in X$ and $g \in G$

$$
\widetilde{c}(g(s), \sigma(g)(t))=j(g, s) \widetilde{c}(s, t) j(\sigma(g), t) .
$$

The following lemma is a preparation for the proof of the theorem.

Lemma 5.1. There exists a unique smooth function $\widetilde{k}$ on $X \times X$ such that

i) $\widetilde{k}\left(o, a_{\theta}\right)=\prod_{j=1}^{r} \cos ^{2} \theta_{j}$, for all $a_{\theta} \in T$.

ii) $\widetilde{k}(k(s)), k(t))=\widetilde{k}(s, t)$, for all $s, t \in X$ and $k \in K$.

Proof. The proof uses several results of harmonic analysis on a compact symmetric space. The function $\ell: a_{\theta} \longmapsto \prod_{j=1}^{r} \cos ^{2} \theta_{j}$ is a smooth function on the torus $T \simeq(\mathbb{R} / \pi \mathbb{Z})^{r}$, which is moreover invariant by the Weyl group ${ }^{3}$. Hence there exists a unique smooth function (still denoted by $\ell$ ) on $X$ which is invariant by $K^{o}$ and coincides on the torus with $\ell$. Now, there exists a unique smooth function $\widetilde{k}$ on $X \times X$ such that $\widetilde{k}(k(s), k(t))=\widetilde{k}(s, t)$ and $\widetilde{k}(o, t)=\ell(t)$ for any $k \in K$ and $s, t \in X$.

\footnotetext{
${ }^{3}$ Recall that the Weyl group consists in permutations of $\{1,2, \ldots, r\}$ and possibly sign changes
} 
To come to the proof of Theorem 5.1, there is clearly a unique possibility for $\widetilde{c}$. Namely, set $\widetilde{c}(s, t)=\widetilde{k}(s, t)^{\frac{p}{2}}$. The function $\widetilde{c}$ is continuous and everywhere nonnegative, as this was already true for $\widetilde{k}$.

To get the covariance relation for $\widetilde{c}$, consider $\mathcal{O}=\kappa(V)$, which is a dense open subset of $X$.

Lemma 5.2. Let $s=\kappa(x)$ and $t=\kappa(y)$ be in $\mathcal{O}$. Then

$$
\widetilde{c}(s, t)=c(x, x)^{-\frac{1}{2}} c(x, y) c(y, y)^{-\frac{1}{2}} .
$$

Proof. Thanks to (21), the RHS of (23) is invariant under the diagonal action of $K$ (at least where defined). As the same is true for the LHS, it is enough to verify the relation when $t=o$, which amounts to $y=0$, and $s=a_{\boldsymbol{\theta}}$ in $T \cap \mathcal{O}$. The corresponding value of $x$ is $x=\sum_{j=1}^{r} \tan \theta_{j} c_{j}$, as $\kappa(x)=a_{\boldsymbol{\theta}}$. Now $c(x, 0)=1$, whereas $c(x, x)=\left(\prod_{j=1}^{r}\left(1+\tan ^{2} \theta_{j}\right)\right)^{p}$. Thus (23) follows.

Lemma 5.3. The function $\widetilde{c}$ satisfies the following covariance property

$$
\widetilde{c}(g(s), \sigma(g)(t))=j(g, s) \widetilde{c}(s, t) j(\sigma(g), t) .
$$

for all $s, t \in X$ and $g \in G$,

Proof. For $g \in G$ and $s, t$ in $\mathcal{O}$ such that $g(s)$ and $g(t)$ are in $\mathcal{O}$, routine calculation starting from the covariance property satisfied by $c$ and using (23) and (21). The full covariance property is then obtained by using the continuity of $\widetilde{c}$ on $X \times X$.

The proof of Theorem 5.1 is then complete.

\section{The domain of convergence for the intertwining integral}

Theorem 6.1. Let $X$ be a simple symmetric $R$-space, of rank $r$ and characteristic numbers $r, a=a_{+}+a_{-}, b, c$ and genus $p=(r-1) a+b+2 c$. Let

$$
c(\lambda)=\int_{X} \widetilde{c}(o, y)^{-\frac{1}{2}+\lambda} d \sigma(y) .
$$

The integral $c(\lambda)$ is absolutely convergent if and only if

$$
\Re(\lambda)>\frac{1}{2}-\frac{c}{p} .
$$


Proof. By use of the integration formula (11), the integral to be checked for convergence is equal to

$$
\begin{gathered}
\int_{[0, \pi]^{r}}\left(\prod_{j=1}^{r} \cos ^{2} \theta_{j}\right)^{\frac{p}{2}\left(-\frac{1}{2}+\lambda\right)} \mid \prod_{i=1}^{r}\left(\sin 2 \theta_{j}\right)^{c-1} \prod_{1 \leq i<j \leq r} \sin \left(\theta_{i}-\theta_{j}\right)^{a_{+}} \ldots \\
\ldots \prod_{1 \leq i<j \leq r} \sin \left(\theta_{i}+\theta_{j}\right)^{a_{-}} \prod_{i=1}^{r}\left(\sin \theta_{j}\right)^{b} \mid d \theta_{1} \ldots d \theta_{r} .
\end{gathered}
$$

Let first assume that $a_{+}=a_{-}=\frac{a}{2}$. Using $\sin (\theta+\varphi) \sin (\theta-\varphi)=$ $\cos ^{2} \theta-\cos ^{2} \varphi$, and $\sin 2 \theta=2 \sin \theta \cos \theta$, the integral $c(\lambda)$ to be checked for convergence is equal to

$$
\int_{[0, \pi]^{r}}\left|\prod_{j=1}^{r} \cos \theta_{j}\right|^{p\left(-\frac{1}{2}+\lambda\right)+c-1}\left(\prod_{j=1}^{r} \sin \theta_{j}\right)^{b+c-1}\left|\prod_{1 \leq i<j \leq r}\left(\cos ^{2} \theta_{i}-\cos ^{2} \theta_{j}\right)\right|^{\frac{a}{2}} d \theta_{1} \ldots d \theta_{r}
$$

The integrand is invariant under the changes $\theta_{j} \mapsto \pi-\theta_{j}$, so that we may integrate on $\left[0, \frac{\pi}{2}\right]^{r}$ and use the change of variables $u_{j}=\cos ^{2} \theta_{j}$ to get (up to a constant)

$$
\int_{[0,1]^{r}} \prod_{j=1}^{r} u_{j}^{\frac{1}{2}\left(p\left(-\frac{1}{2}+\lambda\right)+c\right)-1} \prod_{j=1}^{r}\left(1-u_{j}\right)^{\frac{1}{2}(c+b)-1} \prod_{1 \leq i<j \leq r}\left|u_{i}-u_{j}\right|^{\frac{a}{2}} d u_{1} \ldots d u_{r}
$$

This integral is a special case of the celebrated Selberg's integrals (see e.g. [1] Theorem 8.1.1)

$$
S_{r}(\alpha, \beta, \gamma):=\int_{0}^{1} \ldots \int_{0}^{1} \prod_{i=1}^{r} t_{i}^{\alpha-1}\left(1-t_{i}\right)^{\beta-1} \prod_{1 \leq i<j \leq r}\left|t_{i}-t_{j}\right|^{2 \gamma} d t_{1} \ldots d t_{r} .
$$

The conditions for absolute convergence are

$$
\Re(\alpha)>0, \quad \Re(\beta)>0, \quad \Re(\gamma)>-\min \left\{\frac{1}{r}, \frac{\Re(\alpha)}{r-1}, \frac{\Re(\beta)}{r-1}\right\} .
$$

In the case at hand, the conditions amount to

$$
\Re\left(\frac{1}{2}\left(p\left(-\frac{1}{2}+\lambda\right)+c\right)\right)>0
$$

equivalent to

$$
\Re(\lambda)>\frac{1}{2}-\frac{c}{p}
$$


The constant $c(\lambda)=J_{\lambda} 1$ plays an important role for further analysis of the intertwining operators and the representations $\rho_{\lambda}$. Although we won't use the result, it is worth to give the value of $c(\lambda)$ :

$$
c(\lambda)=C \prod_{j=1}^{r} \frac{\Gamma\left((j-1) \frac{a}{4}-\frac{(r-1) a+b}{4}+\frac{p}{2} \lambda\right)}{\Gamma\left((j-1) \frac{a}{4}+\frac{b+2 c}{4}+\frac{p}{2} \lambda\right)},
$$

where $C$ is a constant which can be made explicit and depends only on $V$.

Let now $V$ be of Euclidean type, so that $a=a_{+}, a_{-}=0, b=0, c=1$, and $p=(r-1) a+2$. The integral to be checked for convergence now reads

$$
c(\lambda)=\int_{[0, \pi]^{r}}\left|\prod_{j=1}^{r} \cos \theta_{j}\right|^{p\left(-\frac{1}{2}+\lambda\right)}\left|\prod_{1 \leq i<j \leq r} \sin \left(\theta_{i}-\theta_{j}\right)\right|^{a} d \theta_{1} \ldots d \theta_{r} .
$$

As the function to be integrated is periodic of period $\pi$ w.r.t. each variable, the integral can be taken over $\left[-\frac{\pi}{2}, \frac{\pi}{2}\right]^{r}$. Then let $t_{j}=\tan \theta_{j}$, and use

$$
\sin ^{2}\left(\theta_{i}-\theta_{j}\right)=\frac{\left(t_{i}-t_{j}\right)^{2}}{\left(1+t_{i}^{2}\right)\left(1+t_{j}^{2}\right)}
$$

to get

$c(\lambda)=\int_{-\infty}^{+\infty} \cdots \int_{-\infty}^{+\infty}\left(\prod_{j=1}^{r}\left(1+t_{j}^{2}\right)\right)^{-\frac{p}{2}\left(\frac{1}{2}+\lambda\right)}\left|\prod_{1 \leq i<j \leq r}\left(t_{i}-t_{j}\right)\right|^{a} d t_{1} d t_{2} \ldots d t_{r}$.

This is again a special case for another version of the Selberg integral (in fact it is the original Selberg formulation, see [8]), which can be seen to converge if and only if $\Re(\lambda)>\frac{1}{2}-\frac{1}{p}$ and is equal to

$$
c \prod_{j=0}^{r-1} \frac{\Gamma\left(p\left(\frac{1}{2}+\lambda\right)-1-(r+j-1) \frac{a}{2}\right)}{\Gamma\left(\frac{p}{2}\left(\frac{1}{2}+\lambda\right)-j \frac{a}{2}\right)^{2}},
$$

where $c$ is an explicit constant depending only on $V$.

From the classification, it remains to consider only the case of the PJTS $V=\mathbb{R}^{p, q}$, where $2 \leq p<q$. For this case, $r=2, a_{+}=q-1, a_{-}=p-1, b=0$ and $c=1$, the genus is equal to $2+(q-1)+(p-1)=p+q=n$. So the integral to be considered is

$$
\int_{0}^{\pi} \int_{0}^{\pi}\left|\sin \left(\theta_{1}-\theta_{2}\right)\right|^{q-1}\left|\sin \left(\theta_{1}+\theta_{2}\right)\right|^{p-1}\left|\cos \theta_{1} \cos \theta_{2}\right|^{n\left(-\frac{1}{2}+\lambda\right)} d \theta_{1} d \theta_{2} .
$$


The integral may become divergent because of the singularities near $\theta_{1}=\frac{\pi}{2}$ or near $\theta_{2}=\frac{\pi}{2}$. This forces the conditions $n\left(-\frac{1}{2}+\Re(\lambda)>-1\right.$, i.e. $\Re(\lambda)>$ $\frac{1}{2}-\frac{1}{n}$. Now if the two conditions are satisfied, it remains to check the convergence for both $\theta_{1}$ and $\theta_{2}$ close to $\frac{\pi}{2}$. Making the change of variables $\varphi_{i}=\theta_{i}-\frac{\pi}{2}$ for $i=1,2$, and using $\sin x \simeq x$ for $x$ close to 0 leads to discussing the convergence near $(0,0)$ of the integral

$$
\iint\left|\varphi_{1}-\varphi_{2}\right|^{q-1}\left|\varphi_{1}+\varphi_{2}\right|^{p-1}\left|\varphi_{1} \varphi_{2}\right|^{n\left(-\frac{1}{2}+\Re(\lambda)\right)} d \varphi_{1} d \varphi_{2}
$$

Using polar coordinates near $(0,0)$, this requires for convergence the (possibly) supplementary condition that

$$
(q-1)+(p-1)+n(-1+2 \Re(\lambda))+1>-1
$$

which amounts to $\Re(\lambda)>0$. Hence the integral converges if and only if $\Re(\lambda)>\frac{1}{2}-\frac{1}{n}$.

\section{$7 \quad$ PHJTS, reduced and non reduced PJTS}

This section is a collection of algebraic/geometric known results on PJTS, mostly without proofs, which will be needed later.

We first discuss the Hermitification process, for which [18] is a good (but sketchy) reference.

Proposition 7.1. Let $V$ be a simple PJTS. Then its Hermitification $\mathbb{V}$ is simple (as a PHJTS), unless $V$ admits a complex structure $J$ for which $V$ is a PHJTS. If this happens, then $\mathbb{V} \simeq V \oplus V_{\text {op }}$, where $V_{\text {op }}$ is the PHJTS $V$ with the opposite complex structure given by $-J$.

Next consider a simple PJTS $V$ and let $\mathbb{V}$ be its Hermitification. The PJTS $V$ is said to be reduced if a primitive tripotent of $V$ is primitive in $\mathbb{V}$. As $\mathbb{V}(c, 1)=\mathbb{C} c$ for a primitive tripotent of $\mathbb{V}$, this is equivalent to the condition that $V(c, 1)=\mathbb{R} c$ for a primitive tripotent of $V$. Notice that if it is true for one primitive tripotent, then it is true for any primitive tripotent. It also amounts to the condition that the characteristic number $c$ of $V$ is equal to 1 .

If $V$ admits a complex structure (that is if $V$ is a PHJTS), then $V$ is not reduced. A PJTS $V$ will be said to be non-reduced if it has no complex structure and is not reduced. Simple PJTS fall into three families : those admitting a complex structure (=PHJTS), the reduced PJTS and the nonreduced PJTS. 
Proposition 7.2. Let $V$ be a simple reduced PJTS of rank $r$. Then its Hermitification $\mathbb{V}$ is a simple PHJTS of rank $r$.

Proposition 7.3. Let $V$ be a simple non-reduced PJTS of rank $r$. Then its Hermitification $\mathbb{V}$ is a simple PHJTS of rank $2 r$. Moreover, given a Jordan frame $c_{1}, c_{2}, \ldots, c_{r}$ of $V$, there exist primitive orthogonal tripotents $d_{1}, d_{2}, \ldots, d_{r}$ in $\mathbb{V}$ such that $c_{j}=d_{j}+\overline{d_{j}}$ and $\left(d_{1}, \overline{d_{1}}, \ldots, d_{r}, \overline{d_{r}}\right)$ is a Jordan frame of $\mathbb{V}$.

For $1 \leq k \leq r$ let $\bar{k}=k+r$ and $d_{\bar{k}}=\overline{d_{k}}$. The Peirce decomposition of $V$ w.r.t. $\left(c_{1}, c_{2}, \ldots, c_{r}\right)$ and the Peirce decomposition of $\mathbb{V}$ w.r.t. $\left(d_{1}, d_{\overline{1}}, \ldots, d_{r}, d_{\bar{r}}\right)$ are connected by the following relations $(1 \leq i<j \leq r)$ :

- $\quad V_{i i}^{\mathbb{C}}=\mathbb{C} d_{i} \oplus \mathbb{C} \overline{d_{i}} \oplus \mathbb{V}_{i \bar{i}}$

- $V_{i j}^{\mathbb{C}}=\mathbb{V}_{i j} \oplus \mathbb{V}_{i \bar{j}} \oplus \mathbb{V}_{\bar{i} j} \oplus V_{\bar{i} \bar{j}}$

- $V_{i 0}^{\mathbb{C}}=\mathbb{V}_{i o} \oplus \mathbb{V}_{\bar{i} 0}$

for $1 \leq i<j \leq r$. The corresponding dimensions (= characteristic numbers) are related by

$$
b=2 b_{\mathbb{C}}, \quad c=2+a_{\mathbb{C}} .
$$

and $a=0$ if $r=1, a=4 a_{\mathbb{C}}$ if $r \geq 2$. Further,

$p=2 c+(r-1) a+b=2\left(2+a_{\mathbb{C}}\right)+(r-1) 4 a_{\mathbb{C}}+2 b_{\mathbb{C}}=2\left(2+(2 r-1) a_{\mathbb{C}}+b_{\mathbb{C}}\right)=2 p_{\mathbb{C}}$.

The last result in this section is a geometric result about the orbits of the structure group of a PJTS.

Proposition 7.4. Let $V$ be a PJTS. Any element $x$ of $V$ is conjugate under $\operatorname{Str}(V)_{0}$ to a tripotent. If $x$ is regular (i.e $L(x, x)$ is invertible), then $x$ is conjugate under $\operatorname{Str}(V)_{0}$ to a maximal tripotent.

Proof. The element $x$ can be written as $x=\sum_{j=1}^{r} t_{j} c_{j}$ for some Jordan frame $\left(c_{1}, c_{2}, \ldots, c_{r}\right)$. As we may change any $c_{j}$ to its opposite, we may assume that $t_{j} \geq 0,1 \leq j \leq r$. Let $J=\left\{j \in\{1,2, \ldots, r\}, t_{j} \neq 0\right\}$. For $j \in J, T_{j}=L\left(c_{j}, c_{j}\right)$ belongs to $\mathfrak{s t r}(V), T_{j} c_{j}=c_{j}$, and for $i \neq j, T_{j} c_{i}=0$. Moreover, the operators $T_{j}$ mutually commute. Hence

$$
\exp \left(-\sum_{j \in J} \log \left(t_{j}\right) T_{j}\right)\left(\sum_{j \in J} t_{j} c_{j}\right)=\sum_{j \in J} c_{j} .
$$

The first statement follows. The second statement corresponds to the case where $J=\{1,2, \ldots, r\}$.

Remark. The orbits of $\operatorname{Str}(V)_{0}$ in a simple PJTS $V$ are described in [6] Part II, Theorem II.2.5. 


\section{The generic minimal polynomial of a PJTS}

\subsection{The construction of the generic minimal polynomial}

Let $\mathbb{K}=\mathbb{R}$ or $\mathbb{C}$. Let $V$ be a PJTS (if $\mathbb{K}=\mathbb{R}$ ) or a PHJTS (if $\mathbb{K}=\mathbb{C}$ ). Let $y$ be in $V$, and define the Jordan product $x_{\cdot y} z$ on $V$ to be

$$
x \cdot y=\{x, y, z\} .
$$

The space $V$ with this product is a Jordan algebra over $\mathbb{K}$, denoted by $V^{(y)}$. The successive powers of the element $x$ for this Jordan product are denoted by $x^{(2, y)}, \ldots, x^{(k, y)}, \ldots$ More generally, if $p$ is a polynomial with vanishing constant term, then $p(x)$ is well defined in $V^{(y)}$. Let $\rho_{y}(x)$ be the supremum of the values of $k$ for which $x, x^{(2, y)}, \ldots, x^{(k, y)}$ are linearly independant, and let $\rho=\sup _{(x, y) \in V} \rho_{y}(x)$ ( $\rho$ is called the absolute rank of $\left.V\right)$. For $x, y \in V \times V$, and $k$ an integer, let $\beta_{k}(x, y)$ be the element of the exterior tensor product $\Lambda^{k} V$ defined by

$$
\beta_{k}(x, y)=x \wedge x^{(2, y)} \wedge \cdots \wedge x^{(k, y)} .
$$

Clearly $\beta_{k}$ is polynomial in $x$ and $y$. For $k \geq \rho+1, \beta_{k}$ vanishes identically on $V \times V$, whereas $\beta_{\rho}$ does not vanish identically on $V \times V$. A couple $(x, y) \in V \times V$ is said to be regular if $\beta_{\rho}(x, y) \neq 0$. Regular elements form a Zariski open dense set in $V \times V$. For $(x, y)$ regular, let $m(T)=m(T, x, y)$ be the monic generator of the ideal in $\mathbb{K}[T]$ of polynomials $p$ such that the polynomial $T p(T)$ annihilates $x$ in $V^{(y)}$.

Proposition 8.1. For $1 \leq j \leq \rho$, there exist (unique) polynomials $m_{j}$ on $\mathbb{K} \times V \times V$ such that, for any regular pair $(x, y)$ in $V \times V$

$$
m(T, x, y)=T^{\rho}+\sum_{j=1}^{\rho}(-1)^{j} m_{j}(x, y) T^{\rho-j} .
$$

The polynomials $m_{j}$ are homogeneous of bidegree $(j, j)$ in $(x, y)$.

See [17] or [6], part V, Proposition IV.3.1.

As a consequence, the RHS of (25) defines a polynomial on $\mathbb{K} \times V \times V$, still denoted by $m(T, x, y)$ and called the generic minimal polynomial of $V$ over $\mathbb{K}$.

The following property is a direct consequence of the construction of the minimal polynomial.

Proposition 8.2. Let $g \in \operatorname{Str}(V)$. Then, for all $x, y \in V$,

$$
m(T, g x, \sigma(g) y)=m(T, x, y),
$$

where $\sigma(g)=\left(g^{t}\right)^{-1}$ if $\mathbb{K}=\mathbb{R}$ and $\sigma(g)=\left(g^{*}\right)^{-1}$ if $\mathbb{K}=\mathbb{C}$. 
The next proposition describes what happens under the Hermitification of a PJTS.

Proposition 8.3. Let $V$ be a PJTS, and let $\mathbb{V}$ be its Hermitification. Then the restriction to $V \times V$ of the generic minimal polynomial (over $\mathbb{C}$ ) of $\mathbb{V}$ is real-valued and coincides with the generic minimal polynomial (over $\mathbb{R}$ ) of $V$.

\subsection{The complex generic minimal polynomial of a PHJTS}

Let us consider the case where $\mathbb{K}=\mathbb{C}$ and $\mathbb{V}$ is a PHJTS.

Proposition 8.4. Let $\mathbb{V}$ be a PHJTS of rank $r$.

i) The absolute rank $\rho$ over $\mathbb{C}$ is equal to $r$.

ii) The generic minimal polynomial $m(T, x, y)$ over $\mathbb{C}$ is holomorphic in $x$ and antiholomorphic in $y$.

iii) Let $\left(c_{1}, c_{2}, \ldots, c_{r}\right)$ be a Jordan frame of $\mathbb{V}$, and let $x=\sum_{j=1}^{r} t_{j} c_{j}, t_{j} \in$ $\mathbb{R}$. Then

$$
m(T, x, x)=\prod_{j=1}^{r}\left(T-t_{j}^{2}\right) .
$$

See [6] Part V, proposition VI.2.6.

\subsection{The generic minimal polynomial of a PJTS}

We now specialize Proposition 8.3 to each of the three types of PJTS.

Proposition 8.5. Let $\mathbb{V}$ be a PHJTS of rank $r$, and let $m(T, x, y)$ be the complex generic minimal polynomial of $\mathbb{V}$. When $\mathbb{V}$ is regarded as a real PJTS, its absolute rank $\rho$ is equal to $2 r$, and the generic minimal polynomial of $\mathbb{V}$ over $\mathbb{R}$ is given by

$$
m_{\mathbb{R}}(T, x, y)=m(T, x, y) \overline{m(T, x, y)} .
$$

Proof. The Hermitification of $\mathbb{V}$ is isomorphic to $\mathbb{V} \oplus \mathbb{V}^{o p}$, and $\mathbb{V}$ is embedded in its Hermitification by the map $x \mapsto(x, x)$. Now the generic minimal polynomial of a sum of two PJTS is easily seen to be the product of the respective generic minimal polynomials. Hence the result.

Proposition 8.6. Let $V$ be a simple reduced PJTS of rank $r$. Then its Hermitification $\mathbb{V}$ is a simple PHJTS of rank $r$ and the absolute rank of $V$ is equal to $r$. 
Proposition 8.7. Let $V$ be a simple non-reduced PJTS of rank $r$. Its Hermitification $\mathbb{V}$ is a simple PHJTS of rank $2 r$ and the absolute rank of $V$ is $2 r$.

\section{The fundamental kernel of a PJTS}

\subsection{The complex fundamental kernel of a PHJTS}

Definition 9.1. Let $\mathbb{V}$ be a PHJTS. Let $m(T, x, y)$ be its complex generic minimal polynomial. Its complex fundamental kernel $h(x, y)$ is defined by

$$
h(x, y)=m(1, x,-y) .
$$

Proposition 9.1. Let $\mathbb{V}$ be a PHJTS of rank $r$ and let $\left(c_{1}, c_{2}, \ldots, c_{r}\right)$ be a Jordan frame of $\mathbb{V}$. The polynomial $h$ satisfies the following properties :

i) $h$ is holomorphic in $x$, antiholomorphic in $y$.

ii) $h(k x, k y)=h(x, y)$ for any $k \in A u t(\mathbb{V})$ and $x, y \in \mathbb{V}$.

iii) for $x=\sum_{j=1} x_{j} c_{j}, y=\sum_{j=1}^{r} y_{j} c_{j}$, where $x_{j}, y_{j} \in \mathbb{C}$

$$
h(x, y)=\prod_{j=1}^{r}\left(1+x_{j} \bar{y}_{j}\right) .
$$

Proof. Only iii) requires some justification. Let $\mathbb{A}$ be the complex span of the $c_{j}$ 's. For $1 \leq j \leq r$, let $e^{i \theta_{j}}$ be any complex number of modulus 1 . Then $\left(e^{i \theta_{1}} c_{1}, e^{i \theta_{2}} c_{2}, \ldots, e^{i \theta_{r}} c_{r}\right)$ is a Jordan frame of $\mathbb{V}$. Hence by Proposition 8.4 iii), for all $t_{1}, t_{2}, \ldots, t_{r} \in \mathbb{R}$,

$$
h\left(\sum_{j=1}^{r} t_{j} e^{i \theta_{j}} c_{j},-\sum_{j=1}^{r} t_{j} e^{i \theta_{j}} c_{j}\right)=\prod_{j=1}^{r}\left(1-t_{j}^{2}\right)=\prod_{j=1}^{r}\left(1+\left(t_{j} e^{\theta_{j}}\right)\left(\overline{-t_{j} e^{\theta_{j}}}\right)\right) .
$$

As the $e^{i \theta_{j}}$ are arbitrary complex numbers of modulus 1, (26) is true for $x=-y \in \mathbb{A}$, hence for all $x, y \in \mathbb{A}$, as both sides of (26) are holomorphic in $x$ and antiholomorphic in $y$.

The next proposition links the complex fundamental kernel and the complex canonical kernel.

Proposition 9.2. Let $\mathbb{V}$ be a simple PHJTS, of rank $r$ and genus $p_{\mathbb{C}}$. Let $c_{\mathbb{C}}(x, y)$ (resp. $h(x, y)$ ) be its complex canonical (resp. fundamental) kernel. Then the following identity holds:

$$
c_{\mathbb{C}}(x, y)=h(x, y)^{p_{\mathbb{C}}} .
$$


For a proof, see [6] Part V, Proposition VI.3.6.

The next results will be needed later when extending results from the PHJTS of tube type to general PHJTS.

Proposition 9.3. Let $\mathbb{V}$ be a simple PHJTS. Let $c$ be a maximal tripotent and let $\mathbb{V}=\mathbb{V}_{2} \oplus \mathbb{V}_{1}$ be the corresponding Peirce decomposition. Then $\mathbb{V}_{2}$ is a simple PHJTS of tube-type.

Proof. The only point to be checked is the simplicity of $\mathbb{V}_{2}$. If $\mathbb{V}$ is of rank 1 , there is nothing to prove. So we may assume that $\mathbb{V}$ has rank $\geq 2$. Suppose $\mathbb{V}_{2}$ would split as $\mathbb{V}_{2}=Y \oplus Z$, a sum of two orthogonal ideals of $\mathbb{V}_{2}$. Let decompose $c=y+z$ with $y \in Y$ and $z \in Z$. From $\{y+z, y+z, y+z\}=y+z$ and the fact that both $Y$ and $Z$ are ideals of $\mathbb{V}_{2}$, one deduces that $y$ and $z$ are orthogonal tripotents. If $y=0$, then $c \in Z$, and hence $L(c, c) t=0$ for any $t$ in $Y$, yielding a contradiction. Hence both $y$ and $z$ are different from 0 . Let further decompose $y$ (resp. $z$ ) as a sum of primitive tripotents of $Y$ (resp. of $Z$ ). A primitive tripotent of $Y$ is a primitive tripotent of $\mathbb{V}_{2}$, and so we would have found two primitive tripotents $d$ in $Y$ and $f$ in $Z$, such that the space $\mathbb{V}\left(d, \frac{1}{2}\right) \cap \mathbb{V}\left(f, \frac{1}{2}\right) \subset Y \cap Z=\{0\}$. But $d$ and $f$ are orthogonal primitive tripotents in $\mathbb{V}$, and hence $\operatorname{dim}\left(\mathbb{V}\left(d, \frac{1}{2}\right) \cap \mathbb{V}\left(f, \frac{1}{2}\right)\right)=a_{\mathbb{C}} \neq 0$ as the rank of $\mathbb{V}_{2}$ is at least 2, thus yielding a contradiction.

Proposition 9.4. Let $\mathbb{V}$ be a simple PHJTS. Let $c$ be a maximal tripotent, $\mathbb{V}_{2} \oplus \mathbb{V}_{1}$ the corresponding Peirce decomposition. Let $h$ (resp. $\left.h^{(2)}\right)$ be the complex fundamental kernel of $\mathbb{V}$ (resp. $\mathbb{V}_{2}$ ). For $x_{2} \in \mathbb{V}_{2}, x_{1} \in \mathbb{V}_{1}$ and $y_{2} \in \mathbb{V}_{2}$

$$
h\left(x_{2}+x_{1}, y_{2}\right)=h\left(x_{2}, y_{2}\right)=h^{(2)}\left(x_{2}, y_{2}\right) .
$$

Proof. The first equality is a consequence of Proposition 4.3 and of Proposition 9.2. For the second equality, observe that the two PHJTS $\mathbb{V}$ and $\mathbb{V}_{2}$ have the same rank, and hence a regular pair $(x, y)$ in $\mathbb{V}_{2}$ is a regular pair in $\mathbb{V}$. So, for such pairs, the minimal polynomial $p_{x, y}$ of $(x, y)$ in $\mathbb{V}_{2}$ is equal to the minimal polynomial $p_{x, y}^{(2)}$ in $\mathbb{V}$. By density, the generic minimal polynomial $m^{(2)}(T, x, y)$ of $\mathbb{V}_{2}$ is equal to the restriction to $\mathbb{V}_{2}$ of the generic minimal polynomial $m(T, x, y)$ of $\mathbb{V}$. The corresponding statement for the complex fundamental kernels follows immediately.

\subsection{The fundamental kernel of a PHJTS}

Let $V$ be a PHJTS, and let $h(x, y)$ be its complex fundamental kernel. Define its (real) fundamental kernel to be

$$
k(x, y)=h(x, y) \overline{h(x, y)} .
$$


Proposition 9.5. Let $V$ be a simple PHJTS, and let $c(x, y)$ (resp. $k(x, y))$ be its canonical (resp. fundamental) kernel. Then

$$
c(x, y)=k(x, y)^{\frac{p}{2}},
$$

where $p$ is the genus (over $\mathbb{R}$ ) of $V$.

Proof. Let $C(x, y)$ be the complex dual Bergman operator of $V$. Recall that the complex canonical kernel is $c_{\mathbb{C}}(x, y)=\operatorname{det}_{\mathbb{C}} C(x, y)$. The canonical kernel (over $\mathbb{R}$ ) is

$$
c(x, y)=\operatorname{det}_{\mathbb{R}} C(x, y)=\operatorname{det}_{\mathbb{C}} C(x, y) \overline{\operatorname{det}_{\mathbb{C}} C(x, y)} .
$$

Hence

$$
c(x, y)=c_{\mathbb{C}}(x, y) \overline{c_{\mathbb{C}}(x, y)}=h(x, y)^{p_{\mathbb{C}}} \overline{h(x, y)}^{p_{\mathbb{C}}}=k(x, y)^{p_{\mathbb{C}}}
$$

The statement follows, as $p=2 p_{\mathbb{C}}$.

\subsection{The fundamental kernel of a reduced PJTS}

Let $V$ be a simple reduced PJTS, and let $\mathbb{V}$ be its Hermitification. Let $h(x, y)$ be the complex fundamental kernel of $\mathbb{V}$. As for the generic minimal polynomial of $\mathbb{V}$, its restriction to the real form $V$ is real valued. Define the fundamental kernel $k$ of $V$ by

$$
k(x, y)=h(x, y)^{2} .
$$

Proposition 9.6. Let $V$ be reduced simple PJTS. Let $c(x, y)(\operatorname{resp} . k(x, y))$ be its canonical (resp. fundamental) kernel. Then, for $x, y \in V$

$$
c(x, y)=k(x, y)^{\frac{p}{2}} .
$$

Proof. In the reduced case, the characteristic numbers of $V$ coincide with the characteristic numbers of $\mathbb{V}$ over $\mathbb{C}$ and $p_{\mathbb{C}}=p$. Hence

$$
c(x, y)=\left|c_{\mathbb{C}}(x, y)\right|=|h(x, y)|^{p_{\mathbb{C}}}=k(x, y)^{\frac{p}{2}} .
$$




\subsection{The fundamental kernel of a non-reduced PJTS}

Let $V$ be a simple non-reduced PJTS. Let $\mathbb{V}$ be its Hermitification, and let $h$ be its complex fundamental kernel.

Lemma 9.1. For all $x, y \in V \times V$,

$$
h(x, y) \geq 0 .
$$

Proof. Let $\left(c_{1}, c_{2}, \ldots, c_{r}\right)$ be a Jordan frame of $V$. Let $x=\sum_{j=1} x_{j} c_{j}$ and $y=\sum_{j=1} y_{j} c_{j}$, where $x_{j}, y_{j} \in \mathbb{R}$. Let $\left(d_{1}, \overline{d_{1}}, \ldots, d_{r}, \overline{d_{r}}\right)$ be an associated Jordan frame of $\mathbb{V}$ as in Proposition 7.3. Then $x=\sum_{j=1}^{r} x_{j} d_{j}+\sum_{j=1}^{r} x_{j} \overline{d_{j}}$ and $y=\sum_{j=1}^{r} y_{j} d_{j}+\sum_{j=1}^{r} y_{j} \overline{d_{j}}$. By Proposition 8.3 and (26),

$$
h(x, y)=\prod_{j=1}^{r}\left(1+x_{j} y_{j}\right)^{2},
$$

hence $h(x, y) \geq 0$. So the property to be proven is true if $x$ and $y$ belong to a common maximal flat subspace of $V$. Now let $c$ be a maximal tripotent in $V$. For any $y$ in $V_{2}(c)$, there exists a Jordan frame $\left(c_{1}, c_{2}, \ldots, c_{r}\right)$ such that $c=c_{1}+c_{2}+\cdots+c_{r}$ and $y=\sum_{j=1}^{r} y_{j} c_{j}$, so $y$ and $c$ belong to a common maximal flat. Hence, $h(c, y) \geq 0$. If now $y$ is arbitrary in $V$, let $y=y_{2}+y_{1}$ where $y_{2} \in V_{2}(c)$ and $y_{1} \in V_{1}(c)$. By Proposition 9.4

$$
h(c, y)=h\left(c, y_{2}+y_{1}\right)=h\left(c, y_{2}\right) \geq 0 .
$$

Hence, for $g \in \operatorname{Str}(V), y \in V$ and any maximal tripotent $c$

$$
h(g c, y)=h(c, \sigma(g) y) \geq 0 .
$$

Thus, if $x$ is regular, Proposition 7.4 shows that $h(x, y) \geq 0$ for any $y \in V$. As the set of regular elements is an open dense set in $V$, the result follows by continuity.

Define the fundamental kernel $k$ of $V$ by

$$
k(x, y)=h(x, y) .
$$

Proposition 9.7. Let $V$ be a non-reduced PJTS. Let $c(x, y)$ (resp. $k(x, y))$ be its canonical (resp. fundamental) kernel. Then, for $x, y \in V$

$$
c(x, y)=k(x, y)^{\frac{p}{2}} .
$$

Proof. Recall that the rank of $\mathbb{V}$ is equal to $2 r$, where $r$ is the rank of $V$, and that $p=2 p_{\mathbb{C}}$ (see Proposition 7.3). Hence, for $x, y$ in $V$,

$$
c(x, y)=\left|c_{\mathbb{C}}(x, y)\right|=h(x, y)^{p_{\mathbb{C}}}=k(x, y)^{\frac{p}{2}} .
$$




\subsection{Résumé}

For any simple PJTS $V$, we defined (by an ad hoc formula depending on the type of the PJTS, see (28), (29), (30)) a fundamental kernel $k(x, y)$ : it is a polynomial on $V \times V$ which is everywhere positive and is related to the canonical kernel $c(x, y)$ by the formula $c(x, y)=k(x, y)^{\frac{p}{2}}$. Observe that the notation is coherent with the notation $\widetilde{k}$ introduced in section 5 .

\section{The Bernstein-Sato identity for the complex fundamental kernel of a PHJTS of tube type}

For a special class of PHJTS, we will need another interpretation of the complex fundamental kernel. Recall that a PHJTS $V$ is said to be of tube type or of Jordan algebra type is there exists a tripotent $c$ such that $V=$ $V_{2}(c)$. If this is the case, the product $x_{\cdot} y=\{x, c, y\}$ endows $V$ with a structure of complex Jordan algebra, with unit element $c$. The map $Q(c)$ is a antiholomorphic involution of $V$ and its fixed points set is a Euclidean Jordan algebra ${ }^{4}$. The converse is true. Let $J$ be a Euclidean Jordan algebra. Let $\mathbb{J}$ be its complexification, and define on $\mathbb{J}$ the trilinear product

$$
\{x, y, z\}=(x \bar{y}) z+(z \bar{y}) x-(x z) \bar{y} .
$$

Then $\mathbb{J}$ is a PHJTS of tube type (called the Hermitification of $J$ ).

The rank of the PHJTS $\mathbb{J}$ coincides with the rank of the Jordan algebra $J$, any Jordan frame of $J$ is a Jordan frame of $\mathbb{J}$. The structure group $\operatorname{Str}(\mathbb{J})$ is the complexification of $\operatorname{Str}(J)$.

Let $J$ be a simple Euclidean Jordan algebra, and denote by $e$ its neutral element. Its Hermitification $\mathbb{J}$ is a simple PHJTS of tube type. Let $\left(c_{1}, c_{2}, \ldots, c_{r}\right)$ be a Jordan frame of $J$. There is a corresponding Peirce decomposition $J=\sum_{j=1}^{r} \mathbb{R} c_{j} \bigoplus_{1 \leq i<j \leq r} J_{i j}$. The spaces $J_{i j}, i<j$ have all the same dimension, usually denoted by $d$. Then $\mathbb{J}=\sum_{j=1}^{r} \mathbb{C} c_{j} \bigoplus_{1 \leq i<j \leq r} \mathbb{J}_{i j}$ is the Peirce decomposition of $\mathbb{J}$ w.r.t. the Jordan frame $\left(c_{1}, c_{2}, \ldots, c_{r}\right)$. Hence the characteristic numbers (over $\mathbb{C}$ ) of $\mathbb{J}$ as a simple PHJTS are $a_{\mathbb{C}}=d$ and $b_{\mathbb{C}}=0$.

Let $\Delta$ be the determinant function of $J$, and extend $\Delta$ as a holomorphic polynomial on $\mathbb{J}$. For $x=\sum_{j=1}^{r} t_{j} c_{j}$, where $\left(c_{1}, c_{2}, \ldots, c_{r}\right)$ is a Jordan frame of $J$

$$
\Delta(x)=\prod_{j=1}^{r} t_{j} .
$$

\footnotetext{
${ }^{4}$ For the theory of Euclidean Jordan algebra, see [5]
} 
Proposition 10.1. Let $q(z, w)$ a polynomial on $\mathbb{J} \times \mathbb{J}$ which is holomorphic in $z$, antiholomorphic in $w$ and satisfies

$$
q(g z, \sigma(g) w)=q(z, w),
$$

for any $g \in S \operatorname{tr}(\mathbb{J})_{0}$. Then there exists a unique polynomial $p$ on $J$, which is invariant by $\operatorname{Aut}(J)_{0}$ such that, for any $x \in J$

$$
q(x, x)=p\left(x^{2}\right) .
$$

For any $x \in J$,

$$
q(x, e)=p(x) .
$$

Moreover, the correspondence $q \longmapsto p$ is one to one.

See [5], Corollary XI.3.4 and Proposition XIV.1.1.

Proposition 10.2. Let $h$ be the complex fundamental kernel of $\mathbb{J}$. For any $x \in \mathbb{J}$ and $1 \leq j \leq r$,

$$
h(x, e)=\Delta(e+x) .
$$

Proof. The polynomial $h$ satisfies all assumptions of the previous proposition. Let $\left(c_{1}, c_{2}, \ldots, c_{r}\right)$ be a Jordan frame of $J$, and let $x=\sum_{j=1}^{r} x_{j} c_{j}$. Then, by (26),

$$
h(x, x)=\prod_{j=1}^{r}\left(1+x_{j}^{2}\right) .
$$

On the other hand, by (31)

$$
\Delta\left(e+x^{2}\right)=\prod_{j=1}^{r}\left(1+x_{j}^{2}\right) .
$$

Hence $h(x, x)=\Delta\left(e+x^{2}\right)$ for any $x \in V$. Thus the polynomial on $\mathbb{J}$ associated to $h$ via Proposition 10.1 is equal to $\Delta(e+x)$. The statement follows.

Let $D=D\left(x, \frac{\partial}{\partial x}\right)$ be a linear differential operator on some open subset $\mathcal{O}$ of $J$ with $\mathcal{C}^{\infty}$ coefficients. Define its symbol as the function $\sigma_{D}$ on $\mathcal{O} \times J$ defined by

$$
D e^{(x, \xi)}=\sigma_{D}(x, \xi) e^{(x, \xi)} .
$$

The symbol is a polynomial in $\xi$, whose coefficients are $\mathcal{C}^{\infty}$ functions of $x$.

Let $g$ be a linear transformation of $J$, and denote by $L(g)$ the left action on $\mathcal{C}^{\infty}(J)$ defined by $L(g) f=f \circ g^{-1}$. A differential operator $D$ is invariant 
under $g$ (i.e. commutes with $L(g))$ if and only if $\sigma_{D}(g x, \sigma(g) \xi)=\sigma_{D}(x, \xi)$, where $\sigma(g)=\left(g^{t}\right)^{-1}$.

Let $\Omega$ be the open cone of squares of $J$ (see [5] for details), and denote by $H$ the connected component of the group $G(\Omega)$ (equivalently of $\operatorname{Str}(J))$. By (the real version of) Proposition 10.1, to each $A u t(J)_{0}$-invariant real-valued polynomial $p$ on $J$, one may associate a unique $H$-invariant differential operator $D=q\left(x, \frac{\partial}{\partial x}\right)$, such that its symbol $\sigma_{D}=$ satisfies $\sigma_{D}(e, \xi)=q(e, \xi)=p(\xi)$.

Consider the homogeneous components of the function $\Delta(e+x)$, i.e.

$$
\Delta(e+x)=\sum_{k=0}^{r} a_{k}(x)
$$

where $a_{k}$ is a homogeneous polynomial on $J$ of degree $k$. Clearly the $a_{k}^{\prime} s$ are invariant by $A u t(J)$. Denote by $M_{k}$ the $H$-invariant differential operator on $J$ such that

$$
\sigma_{M_{k}}(e, \xi)=a_{k}(\xi) .
$$

(cf [5] ch. XIV). Although we won't use the result, notice that the $M_{k}$ generate the algebra of $H$-invariant differential operators on $J$.

A large family of $H$-invariant differential operators on $J$ is provided by the following result (see [5] Proposition XIV.1.5). Recall that $\Delta(x)>0$ for $x \in \Omega$.

Proposition 10.3. Let $s$ be a complex number, and let $D_{s}$ be the differential operator defined (a priori on $\Omega$ ) by

$$
D_{s}=\Delta(x)^{1+s} \circ \Delta\left(\frac{\partial}{\partial x}\right) \circ \Delta(x)^{-s} .
$$

Then

i) $D_{s}$ extends to a differential operator on $J$ with polynomial coefficients.

ii) $D_{s}$ is $H$-invariant.

iii)

$$
D_{s}=\sum_{k=0}^{r}(-1)^{k} \prod_{j=1}^{k}\left(s-(j-1) \frac{d}{2}\right) M_{r-k} .
$$

See [5] ch. XIV.

Let $\iota$ be the inversion on $\Omega$ defined by $\iota(x)=x^{-1}$. Whenever $D$ is a $H$-invariant operator on $\Omega$, then the differential operator $D^{\iota}$ defined by

$$
D^{\iota} f=(D(f \circ \iota)) \circ \iota
$$


can be shown to be also $H$-invariant. In particular, we have the following result :

$$
D_{s}^{\iota}=(-1)^{r} D_{t},
$$

where $t=\frac{d}{2}(r-1)-s$. See [5] proposition XIV.1.8.

Recall the Bernstein-Sato identity for the determinant polynomial of the Euclidean Jordan algebra $J$ (see [5] Prop. VII.1.4) .

Proposition 10.4. Let $s$ be a complex number. For $x$ in $\Omega$

$$
\Delta\left(\frac{\partial}{\partial x}\right) \Delta^{s}(x)=b(s) \Delta(x)^{s-1}
$$

where

$$
b(s)=b_{r, d}(s)=s\left(s+\frac{d}{2}\right) \ldots\left(s+(r-1) \frac{d}{2}\right) .
$$

Proposition 10.5. For $s$ in $\mathbb{C}$, let $s^{*}=s+\frac{d}{2}(r-1)$. Then, for $x$ in $\Omega$

$$
(-1)^{r} D_{s^{*}}\left(\Delta(e+x)^{s}\right)=b(s) \Delta(e+x)^{s-1} .
$$

Proof. Using the invariance of $\Delta\left(\frac{\partial}{\partial x}\right)$ under translations, for $x \in \Omega$,

$$
\Delta\left(\frac{\partial}{\partial x}\right) \Delta(e+x)^{s}=b(s) \Delta(x+e)^{s-1} .
$$

Recall the identity, valid for $x$ invertible (in particular for $x$ in $\Omega$ ) :

$$
\Delta(e+x)=\Delta(x) \Delta\left(e+x^{-1}\right)
$$

Hence

$$
\Delta(x)^{-s+1} \Delta\left(\frac{\partial}{\partial x}\right)\left(\Delta(x)^{s} \Delta\left(e+x^{-1}\right)^{s}\right)=b(s) \Delta(e+x)^{s-1} \Delta(x)^{-s+1} .
$$

Setting for a while $d_{s}(x)=\Delta(e+x)^{s}$, the last identity can be rewritten as

$$
D_{-s}\left(d_{s} \circ \iota\right)(x)=b(s) d_{s-1}\left(x^{-1}\right)
$$

Using (33), this can be rewritten as

$$
(-1)^{r} D_{s^{*}}\left(d_{s}(x)\right)=b(s) d_{s-1}(x),
$$

which, up to notation agrees with (36). 
The advantage of this new Bernstein-Sato identity is that now the differential operator involved is invariant under $H$. For sake of simplicity, let $E_{s}$ be the differential operator with polynomials coefficients on $V$ defined by

$$
E_{s}=(-1)^{r} D_{s^{*}}=\sum_{k=0}^{r}(-1)^{r-k}\left(\prod_{j=1}^{k}\left(s+\frac{d}{2}(r-j)\right) M_{r-k} .\right.
$$

Recall the connection between $\Delta$ and the complex canonical kernel $h$ of the Hermitification $\mathbb{J}$ of $J$ (see proposition 10.1).

Lemma 10.1. Let $x, y$ in $\Omega$. Then

$$
h(x, y)>0 .
$$

Proof. As $h(e, y)=\Delta(e+y)$ for any $y \in J, h(e, y)>0$ for any $y \in \Omega$. Let $g$ be in $H$. Then by invariance of $h$ under the action of $H, h\left(g e,\left(g^{t}\right)^{-1} y\right)>0$ for any $y$ in $\Omega$, hence $h(g e, y)>0$ for any $y \in \Omega$. As $g$ runs through $H$, ge runs through all of $\Omega$. Hence the positivity of $h$ on $\Omega \times \Omega$.

Proposition 10.6. For $x, y$ in $\Omega$,

$$
E_{s}\left(x, \frac{\partial}{\partial x}\right) h(x, y)^{s}=b(s) h(x, y)^{s-1} .
$$

Proof. Let $y$ be in $\Omega$. As $H$ is transitive on $\Omega$, choose $g \in H$ such that $\sigma(g) e=y$. Then $h(x, y)=h(x, \sigma(g) e)=h\left(g^{-1} x, e\right)$. Further, by the invariance of $E_{s}$ under the action of $H$

$$
\begin{gathered}
E_{s} h(x, y)^{s}=E_{s} h\left(g^{-1} x, e\right)^{s} \\
=\left(E_{s} h(., e)^{s}\right)\left(g^{-1} x\right)=b(s) h\left(g^{-1} x, e\right)^{s-1}=b(s) h(x, y)^{s-1} .
\end{gathered}
$$

We now extend this Bernstein-Sato identity to $\mathbb{J}$.

If $D$ is any differential operator on a real vector space $E$ with polynomial coefficients, we extend it as a holomorphic differential operator $\mathbb{D}$ with holomorphic polynomial coefficients on the complexification $\mathbb{E} \otimes_{\mathbb{R}} \mathbb{C}$ as follows : choose coordinates on $E$, and to $D=x^{\beta} \frac{\partial^{|\alpha|}}{\partial x^{\alpha}}$, where $\alpha$ and $\beta$ are multiindices, associate $\mathbb{D}=z^{\beta} \frac{\partial^{|\alpha|}}{\partial z^{\alpha}}$, and extend this correspondence linearly. The extension does not depend on the choice of the coordinates on $E$. It 
satisfies the restriction principle : for a holomorphic function $f$ defined on a neighborhood $\mathcal{O}$ of some point $x$ in $E$,

$$
\mathbb{D} f(x)=D\left(f_{\mid \mathcal{O} \cap E}\right)(x) \text {. }
$$

The symbol $\sigma_{\mathbb{D}}$ of a holomorphic differential operator $\mathbb{D}$ is defined by

$$
\mathbb{D}\left(z, \frac{\partial}{\partial z}\right) e^{(z, \zeta)}=\sigma_{\mathbb{D}}(z, \zeta) e^{(z, \zeta)},
$$

where the inner product on $J$ is extended to a Hermitian form on $\mathbb{J}$. The symbol is holomorphic in $z$ and is an antiholomorphic polynomial is $\zeta$. If $\mathbb{D}$ is the holomorphic extension of a differential operator $D$ on $E$, then $\sigma_{\mathbb{D}}$ is the extension of $\sigma_{D}$ which is holomorphic in $z$ and antiholomorphic in $\zeta$.

Apply the procedure of holomorphic extension to the operators $E_{s}$ and $M_{k}$ to obtain a holomorphic differential operators $\mathbb{E}_{s}$ and $\mathbb{M}_{k}$ on $\mathbb{J}$.

Theorem 10.1. Let $z, w$ be in $\mathbb{J} \times \mathbb{J}$, and assume that $h(z, w) \neq 0$. The following identity holds :

$$
\mathbb{E}_{s}\left(z, \frac{\partial}{\partial z}\right) h(z, w)^{s}=b(s) h(z, w)^{s-1},
$$

provided $h(z, w)^{s}$ and $h(z, w)^{s-1}$ are computed from the same local determination of $\log h(z, w)$.

Proof. Let

$$
\mathcal{O}=\{(z, w) \in \mathbb{J} \times \mathbb{J}, h(z, w) \neq 0\} .
$$

As $h(z, w)$ is a holomorphic polynomial on $\mathbb{J} \times \mathbb{J}^{o p}, \mathcal{O}$ is a pathwise connected open subset of $\mathbb{J} \times \mathbb{J}$, which contains $\Omega \times \Omega$. This allows to analyticaly continue the identity (38) to $\mathcal{O}$ for each choice of a determination of $\log h(z, w)$. Hence the result.

The identity (40) is the Bernstein-Sato identity for the complex fundamental kernel of $\mathbb{J}$, that is to say for a PHJTS of tube-type.

\section{Bernstein-Sato identity for the complex funda- mental kernel of a PHJTS}

Let $\mathbb{V}$ be a simple PHJTS. Let $h$ be the complex fundamental kernel of $\mathbb{V}$. As a consequence of the covariance properties of $h, h\left(t x, t^{-1} y\right)=h(x, y)$ for 
any $t \in \mathbb{R}^{*}$, so that the decomposition in homogeneous components of $h$ is of the form

$$
h(x, y)=\sum_{k=0}^{r} h_{k}(x, y),
$$

where $h_{k}$ is homogeneous of bidegree $k$ in $x$ and $y$. The $h_{k}$ 's inherit from $h$ the following properties.

Proposition 11.1. For $0 \leq k \leq r$,

i) $h_{k}$ is holomorphic in $x$ and antiholomorphic in $y$.

ii) for $g \in \operatorname{Str}(\mathbb{V}), \quad h_{k}(g x, \sigma(g) y)=h_{k}(x, y)$.

Let $c$ be a maximal tripotent of $\mathbb{V}$. Let $\mathbb{V}=\mathbb{V}_{2} \oplus \mathbb{V}_{1}$ be the corresponding Peirce decomposition of $\mathbb{V}$. The space $\mathbb{V}_{2}$ is a sub-PHJTS which is of tubetype. It has a natural structure of complex Jordan algebra by setting

$$
x \cdot c y=\{x, c, y\}
$$

The map $Q(c)$ preserves $\mathbb{V}_{2}$ and $J=\left\{x \in \mathbb{V}_{2}, Q(c) x=x\right\}$ is a real form of $\mathbb{V}_{2}$ is a simple Euclidean Jordan algebra (see Proposition 9.3).

Let $h^{(2)}$ be the complex fundamental kernel of $\mathbb{V}_{2} \simeq \mathbb{J}$, and for $0 \leq$ $k \leq r$, let $h_{k}^{(2)}$, be its homogeneous components. The next statement is a consequence of Proposition 9.4.

Proposition 11.2. For $x_{2}, y_{2} \in \mathbb{V}_{2}$ and $x_{1} \in \mathbb{V}_{1}$,

$$
h_{k}\left(x_{2}+x_{1}, y_{2}\right)=h_{k}\left(x_{2}, y_{2}\right)=h_{k}^{(2)}\left(x_{2}, y_{2}\right) .
$$

The invariance of the polynomial $h_{k}$ under $\operatorname{Str}(\mathbb{V})$ allows to associate a holomorphic differential operator $M_{k}$ with polynomial coefficients on $\mathbb{V}$, such that its symbol satisfies $\sigma_{M_{k}}(x, \xi)=h_{k}(x, \xi)$. Similarly, let $M_{k}^{(2)}$ be the differential operator on $\mathbb{V}_{2}$ associated to the $h_{k}^{(2)}$. Notation is coherent in the sense that our present $M_{k}^{(2)}$ was denoted by $M_{k}$ in section 10.

Proposition 11.3. Then, for any function $f$ in $\mathcal{C}^{\infty}(\mathbb{V})$ and $x_{2} \in \mathbb{V}_{2}$,

$$
M_{k} f\left(x_{2}\right)=M_{k}^{(2)}\left(f_{\mid \mathbb{V}_{2}}\right)\left(x_{2}\right) .
$$

Proof. Let $\xi=\xi_{2}+\xi_{1}$ be in $\mathbb{V}_{2}$. The symbol $h_{k}\left(x_{2}, \xi\right)$ at $x_{2}$ does depend only on $\xi_{2}$, and is equal to $h_{k}^{(2)}\left(x_{2}, \xi_{2}\right)$. Hence the action of the differential operator $M_{k}$ at $x_{2}$ uses only differentiations in the directions of $\mathbb{V}_{2}$. The statement follows. See similar results in [6], part III, section VI.4.4. 
With (37) in mind, and recalling that the parameter $d$ of the Jordan algebra point of view coincides with $a_{\mathbb{C}}$, set the following definition :

$$
\mathbb{E}_{s}=\sum_{k=0}^{r}(-1)^{r-k}\left(\prod_{j=1}^{k}\left(s+\frac{a_{\mathbb{C}}}{2}(r-j)\right) M_{r-k} .\right.
$$

Theorem 11.1. Let $z, w$ be in $\mathbb{V} \times \mathbb{V}$, and assume that $h(z, w) \neq 0$. The following identity holds :

$$
\mathbb{E}_{s}\left(z, \frac{\partial}{\partial z}\right) h(z, w)^{s}=b(s) h(z, w)^{s-1},
$$

where $h(z, w)^{s}$ and $h(z, w)^{s-1}$ are computed from the same local determination of $\log h(z, w)$ and $b=b_{r, a_{\mathbb{C}}}(c f(35))$.

Proof. Let $c$ be a maximal tripotent of $\mathbb{V}$, and let $\mathbb{V}=\mathbb{V}_{2} \oplus \mathbb{V}_{1}$ be the associated decomposition of $\mathbb{V}$. For an appropriate choice of $c$, we may assume (and hence do assume) that $w=w_{2} \in \mathbb{V}_{2}$. The function $h\left(z, w_{2}\right)$ depends only on the component $z_{2}$ of $z$ and the restriction $h_{\mid \mathbb{V}_{2} \times \mathbb{V}_{2}}$ coincide with the complex fondamental kernel $h^{(2)}$ of $\mathbb{V}_{2}$. Now $\mathbb{E}_{s}$ is a linear combination of the differential operators $M_{k}$. By Proposition 11.3, we are reduced to a computation inside $\mathbb{V}_{2}$. Now use the Bernstein-Sato identity (40) for $\mathbb{V}_{2} \simeq \mathbb{J}$ to conclude.

\section{Bernstein-Sato identities for the fundamental kernel of a PJTS}

\subsection{Berstein-Sato identity for the fundamental kernel of a PHJTS}

Let $V$ be a simple PHJTS. Let $h(x, y)$ be its complex fundamental kernel. Then (cf (28)), as a real PJTS, its fundamental kernel is given by

$$
k(x, y)=|h(x, y)|^{2} .
$$

Let $D=\sum_{\alpha} a_{\alpha}(z) \frac{\partial^{|\alpha|}}{\partial z^{\alpha}}$ be a holomorphic differential operator on $V$. Denote by $\bar{D}$ the (antiholomorphic) differential operator given by

$$
\bar{D}=\sum_{j=1}^{k} \overline{a_{\alpha}}(z) \frac{\partial^{|\alpha|}}{\partial \bar{z}^{\alpha}} .
$$


Then, for any smooth function on $V, \bar{D}(\bar{f})=\overline{D f}$.

Let $\mathbb{E}_{s}$ be the holomorphic differential operator defined on $V$ (viewed as a PHJTS) by (37) and let $b(s)=b_{r, a_{\mathbb{C}}}(s)$ be defined by (35).

Proposition 12.1. Let $x, y \in V$ and assume that $k(x, y) \neq 0$. Then

$$
\mathbb{E}_{s} \overline{\mathbb{E}_{s}} k(x, y)^{s}=b(s)^{2} k(x, y)^{s-1} .
$$

Proof. Near a point $(x, y)$ such that $h(x, y) \neq 0$, choose a local determination of $\log h(x, y)$, and consider the corresponding local determinations for the complex powers of $h$, such as $h(x, y)^{s}$. Now, $\overline{\left(h(x, y)^{\bar{s}}\right)}$ is a determination of $(\overline{h(x, y)})^{s}$ such that

$$
k(x, y)^{s}:=e^{s \log k(x, y)}=h(x, y)^{s} \overline{h(x, y)^{\bar{s}}} .
$$

Hence

$$
\begin{gathered}
\left(\mathbb{E}_{s} \overline{\mathbb{E}_{s}}\right) k(x, y)^{s}=\mathbb{E}_{s} h(x, y)^{s} \overline{\mathbb{E}_{s}} \overline{h(x, y)^{\bar{s}}} \\
=b(s) \overline{b(\bar{s})} h(x, y)^{s-1} \overline{h(x, y)^{\bar{s}-1}}=b(s)^{2} k(x, y)^{s-1} .
\end{gathered}
$$

\subsection{Bernstein-Sato identity for a reduced PJTS}

Let $V$ be a simple reduced PJTS, and let $\mathbb{V}$ its Hermitification, which is a simple PHJTS. Let $h$ be the complex fundamental kernel of $\mathbb{V}$. Recall (cf (29)) that its fundamental kernel $k$ is given by

$$
k(x, y)=h(x, y)^{2} .
$$

Then $k$ is a polynomial function which is nonegative on $V \times V$.

With again (37) in mind, let $E_{s}$ be the differential operator on $V$ defined by

$$
E_{s}=\sum_{k=0}^{r}(-1)^{r-k}\left(\prod_{j=1}^{k}\left(s+\frac{a}{2}(r-j)\right) M_{r-k},\right.
$$

where $r$ is the rank and $a$ the first characteristic number of $V$. Then the complexified holomorphic differential operator of $E_{s}$ coincides with the holomorphic differential operator $\mathbb{E}_{s}$ on $\mathbb{V}$ by $(41)$, as $a_{\mathbb{C}}=a$.

Let $t \in \mathbb{R}, t \neq 0$. For $t>0$, we choose the usual determination of $\log t$ and the corresponding determination of $t^{s}$. When $t<0$, we use $\log t=$ $\log (-t)+i \pi$, so that the corresponding determinaton of $t^{s}$ is $t^{s}=(-t)^{s} e^{i \pi s}$. With these notations, observe that $t^{2 s}=\left(t^{2}\right)^{s}$ for $t>0$, and $t^{2 s}=e^{2 i \pi s}\left(t^{2}\right)^{s}$ when $t<0$. 
Proposition 12.2. Let $x, y \in V$ such that $k(x, y) \neq 0$. Then

$$
\left(E_{2 s-1} \circ E_{2 s}\right) k(x, y)^{s}=b(2 s) b(2 s-1) k(x, y)^{s-1},
$$

where $b=b_{r, a}($ see $(35))$.

Proof. By applying twice the Bernstein-Sato identity on $\mathbb{V}$ for the values $2 s$ and $2 s-1$, we get

$$
\left(\mathbb{E}_{2 s-1} \circ \mathbb{E}_{2 s}\right) h(x, y)^{2 s}=b(2 s) b(2 s-1) h(x, y)^{2 s-2},
$$

with $b=b_{r, a}$. Now restrict this identity to $V$ by the restriction principle. When $h(x, y)>0$, this clearly implies the statement. When $h(x, y)<0$, oberve that $h(x, y)^{2 s}=e^{-2 i \pi s} k(x, y)^{2}$, whereas $h(x, y)^{2 s-2}=e^{-2 i \pi(s-1)} k(x, y)^{s}$, so that the statement also holds.

\subsection{Berstein-Sato identity for a non reduced PJTS}

Let $V$ be a simple non reduced PJTS. Let $\mathbb{V}$ be its Hermitification, which is a simple PHJTS. Let $h(x, y)$ be the complex fundamental kernel of $\mathbb{V}$. Recall (cf (30)) that the fundamental kernel $k$ of $V$ is defined by $k(x, y)=h(x, y)$ and is everywhere $\geq 0$ on $V \times V$.

Let

$$
E_{s}=\sum_{k=0}^{2 r}(-1)^{2 r-k} \prod_{j=1}^{k}\left(s+\frac{c-2}{2}(2 r-j)\right) M_{2 r-k} .
$$

Then its complexification $\mathbb{E}_{s}$ coincides with the operator defined (under the same notation) for the PHJTS $\mathbb{V}$ by (41), as $\mathbb{V}$ has rank $2 r$ and characteristic number $a_{\mathbb{C}}=c-2(\mathrm{cf}$ Proposition 7.3).

Proposition 12.3. Let $x, y$ in $V$ and assume that $k(x, y) \neq 0$. Then

$$
E_{s} k(x, y)^{s}=b(s) k(x, y)^{s-1} .
$$

where $b=b_{2 r, c-2}$.

Proof. Apply the restriction rule (39) to the Berstein-Sato identity (40) on $\mathbb{V}$. 


\section{Meromorphic continuation of the intertwining operators}

Let $f$ be in $\mathcal{C}^{\infty}(X)$. The intertwining operator is given by

$$
J_{\lambda} f(s)=\int_{X} \widetilde{c}(s, t)^{-\frac{1}{2}+\lambda} f(t) d \sigma(t) .
$$

We want to prove that $\lambda \longmapsto J_{\lambda}(f)(x)$ can be continued meromorphically to $\mathbb{C}$.

Observe that, for any $k \in K \rho_{\lambda}(k)$ is the left translation by $k$, and similarly for $\rho_{-\lambda}^{\sigma}(k)$. So $J_{\lambda}$ commutes to the left translation by elements of $K$. Hence the meromorphic continuation, if valid for a function $f$, is also valid for any $K$-translate of $f$. Let $\mathcal{O}$ be a (small) neigborhood of $o$ in $X$. By a partition of unity argument, any function $f$ in $\mathcal{C}^{\infty}(X)$ can be written as a finite sum of $K$-translates of functions which have their supports contained in $\mathcal{O}$. So it is enough to prove the meromorphic continuation for functions having their support contained in $\mathcal{O}$. This remark allows to transfer the problem of meromorphic continuation from the compact picture to the noncompact picture.

Denote by $\mathcal{C}^{\infty}(X, \mathcal{O})$ the space of smooth functions which have their support contained in $\mathcal{O}$. Let $\iota_{\lambda}$ be the map defined on $\mathcal{C}^{\infty}(X, \mathcal{O})$ by

$$
\iota_{\lambda} f(x)=\left(c(x, x)^{-\frac{1}{2}}\right)^{\frac{1}{2}+\lambda} f(\kappa(x)) .
$$

in accordance with the way densities should transform under the variable change $s=\kappa(x)$.

Proposition 13.1. For any $f \in \mathcal{C}^{\infty}(X, \mathcal{O})$,

$$
\iota_{-\lambda}\left(J_{\lambda} f\right)(x)=\int_{V} c(x, y)^{-\frac{1}{2}+\lambda}\left(\iota_{\lambda} f\right)(y) d y .
$$

Proof.

$$
\begin{gathered}
\iota_{-\lambda}\left(J_{\lambda} f\right)(x)=\left(c(x, x)^{-\frac{1}{2}}\right)^{\frac{1}{2}-\lambda} \int_{S} \widetilde{c}(\kappa(x), t)^{-\frac{1}{2}+\lambda} f(t) d \sigma(t) \\
=\left(c(x, x)^{-\frac{1}{2}}\right)^{\frac{1}{2}-\lambda} \int_{V} \widetilde{c}(\kappa(x), \kappa(y))^{-\frac{1}{2}+\lambda} f(\kappa(y)) c(y, y)^{-\frac{1}{2}} d y \\
=\left(c(x, x)^{\frac{1}{2}}\right)^{-\frac{1}{2}+\lambda} \int_{V} \widetilde{c}(\kappa(x), \kappa(y))^{-\frac{1}{2}+\lambda}\left(\iota_{\lambda} f\right)(y)\left(c(y, y)^{\frac{1}{2}}\right)^{-\frac{1}{2}+\lambda} d y
\end{gathered}
$$




$$
=\int_{V} c(x, y)^{-\frac{1}{2}+\lambda}\left(\iota_{\lambda} f\right)(y) d y,
$$

where we used the change of variable $t=\kappa(y)$ first, and then (23).

As the map $\iota_{\lambda} f$ depends holomorphically on $\lambda$ on $\mathbb{C}$, the meromorphic continuation of $J_{\lambda} f$ can be deduced, by a routine argument from the meromorphic continuation of

$$
I_{\lambda} f(x)=\int_{V} c(x, y)^{-\frac{1}{2}+\lambda} f(y) d y
$$

where $f$ is a function in $\mathcal{C}_{c}^{\infty}(V)$.

In order to describe the meromorphic continuation, it is more convenient to work with the meromorphic continuation (in $s$ ) of the integral

$$
J_{s}=J_{s} f(x)=\int_{V} k(x, y)^{s} f(y) d y,
$$

the two problems being related by the equality

$$
I_{\lambda} f=J_{s} f, \quad \text { for } s=-\frac{p}{4}+\frac{p \lambda}{2} .
$$

By theorem 6.1, the integral $J_{s}$ is absolutely convergent if $\Re s>-\frac{c}{2}$. Now the various Bernstein identities may be written as

$$
B_{s}\left(x, \frac{\partial}{\partial x}\right) k(x, y)^{s}=b(s) k(x, y)^{s-1}
$$

where $B_{s}$ is a differential operator with polynomial coefficients, and $b$ is polynomial in $s$. Hence, by an integration by parts,

$$
J_{s-1}(f)=b(s)^{-1} \int_{V} k(x, y)^{s}\left(B_{s}^{t} f\right)(y) d y=b(s)^{-1} J_{s}\left(B_{s}^{t} f\right) .
$$

This can be used to define the meromorphic continuation near a point $s-1$ provided $s$ is not a pole of $J_{s}$ and $b(s) \neq 0$.

For $s=-\frac{c}{2}$, the integral $J_{s} 1$ studied in section 7 is not convergent. As the integrand is a nonnegative function, it really means that $-\frac{c}{2}$ is a pole of $J_{s}$. Then $1-\frac{2}{c}$ must be a zero of $b$. The set of poles is the union of the "left integral half lines" $\{\alpha-k, k=1,2 \ldots\}$ where $\alpha$ runs through the zeroes of $b$ such that $\alpha-1 \leq \frac{c}{2}$. 


\subsection{Meromorphic continuation for a PHJTS}

Let $V$ be a simple PHJTS. The characteristic number $c$ in this case is equal to 2 , so that the integral $J_{s}$ is absolutely convergent if $\Re s>-1$. Let $a_{\mathbb{C}}$ be the complex dimension of $V_{i j}$ for $i \neq j$.

Theorem 13.1. Let $V$ be a simple PHJTS. The integral $J_{s}$ has a meromorphic extension to $\mathbb{C}$ with poles at

- $-1,-2, \ldots,-k, \ldots$ if $r=1$ or if $r \geq 2$ and $a_{\mathbb{C}}$ is even.

- $-1,-2, \ldots,-k, \ldots$ and $-1-\frac{a_{\mathbb{C}}}{2}, \ldots,-k-\frac{a_{\mathbb{C}}}{2}$, if $a_{\mathbb{C}}$ is odd.

Proof. The Bernstein polynomial $b$ is given by $b(s)=b_{r, a_{\mathbb{C}}}(s)^{2}$ and its set of zeroes is

$$
\left\{0,-\frac{a_{\mathbb{C}}}{2}, \ldots,-(r-1) \frac{a_{\mathbb{C}}}{2}\right\} .
$$

The result follows.

\subsection{Meromorphic continuation for a reduced PJTS}

Theorem 13.2. Let $V$ be a simple reduced PJTS. The integral $J_{s}$ has a meromorphic extension to $\mathbb{C}$ with poles at

- $-\frac{1}{2},-1,-\frac{3}{2}, \ldots,-\frac{k}{2}, \ldots$ if $r=1$ or if $r \geq 2$ and $a$ is even.

- $-\frac{1}{2},-1,-\frac{3}{2}, \ldots,-\frac{k}{2}, \ldots$ and $-\frac{a}{4}-\frac{1}{2}, \ldots,-\frac{a}{4}-\frac{k}{2}, \ldots$ if $a$ is odd.

Proof. In this case, $c=1$, so that the integral $J_{s}$ converges absolutely for $\Re s>-\frac{1}{2}$. The Bernstein polynomial $b$ is given by

$$
b(s)=b_{r, a}(2 s) b_{r, a}(2 s-1)
$$

The zeroes of $b(2 s) b(2 s-1)$ are are

$$
\left\{0,-\frac{a}{4}, \cdots-(r-1) \frac{a}{4}\right\} \cup\left\{\frac{1}{2}, \frac{1}{2}-\frac{a}{4}, \ldots, \frac{1}{2}-(r-1) \frac{a}{4}\right\} .
$$

The result follows.

\subsection{Meromorphic continuation for the non reduced PJTS}

Theorem 13.3. Let $V$ be a simple non reduced PJTS. Then the integral $J_{s}$ has a meromorphic extension to $\mathbb{C}$ with poles at

- $-\frac{c}{2},-\frac{c}{2}-1, \ldots,-\frac{c}{2}-k, \ldots$ if $r=1$ or if $r \geq 2$ and $c$ is even

- $-\frac{c}{2},-\frac{c}{2}-1, \ldots,-\frac{c}{2}-k, \ldots$ and $-c+1,-c,-c-1, \ldots,-c-k, \ldots$ if $r \geq 2$ and $c$ is odd. 
Proof. In this case, the first pole is at $-\frac{c}{2}$, and the polynomial $b$ is equal to $b_{2 r, c-2}$ which has for zeroes

$$
\left\{0,-\frac{c}{2}+1, \ldots,(2 r-1)\left(-\frac{c}{2}+1\right\},\right.
$$

As $c \geq 3,-1>-\frac{c}{2}$ does not match the condition to be a pole. The second zero of $b$ corresponds to the first pole and produces the first family of poles. If $r=1$ this exhausts the possibilites, but if $r \geq 2$ and $c$ is odd, the third zero of $b$ contributes to a second family of poles.

\section{Annex : a classification of the simple PJTS}

Table 1

Simple PHJTS and compact Hermitian symmetric spaces

$\begin{array}{rlllll} & \mathbb{V} & \mathbb{X} & r & a_{\mathbb{C}} & b_{\mathbb{C}} \\ s \geq 1 & \mathbb{C}^{1 \times s} & \mathbb{P}_{s}(\mathbb{C}) & 1 & 0 & s-1 \\ 2 \leq r \leq s & \mathbb{C}^{r \times s} & G r\left(r, \mathbb{C}^{r+s}\right) & r & 1 & s-r \\ r \geq 3 & \operatorname{Asym}(2 r, \mathbb{C}) & S O(4 r) / U(2 r) & r & 2 & 0 \\ r \geq 2 & \operatorname{Asym}(2 r+1, \mathbb{C}) & S O(4 r+2) / U(2 r+1) & r & 2 & 2 \\ r \geq 2 & \operatorname{Sym}(r, \mathbb{C}) & S p(r) / U(r) & r & 1 & 0 \\ n \geq 5 & \mathbb{C}^{n} & \mathbb{Q}^{n}(\mathbb{C}) & 2 & n-2 & 0 \\ & \mathbb{O}_{\mathbb{C}}^{1 \times 2} & E_{6} / \operatorname{Spin}(10) \cdot T & 2 & 6 & 4 \\ & \operatorname{Herm}\left(2, \mathbb{O}_{\mathbb{C}}\right) & E_{7} / E_{6} \cdot T & 3 & 8 & 0\end{array}$

Table 2

Simple reduced PJTS of Euclidean type 


$\begin{array}{lllll} & V & X & r & a \\ & \mathbb{R} & S^{1} & 1 & 0 \\ r \geq 2 & \operatorname{Sym}(r, \mathbb{R}) & S O(2 r) / U(r) & r & 1 \\ r \geq 2 & \operatorname{Herm}(r, \mathbb{C}) & U(r) & r & 2 \\ r \geq 2 & \operatorname{Herm}(r, \mathbb{H}) & U(2 r) / S p(r) & r & 4 \\ n \geq 4 & \mathbb{R}^{1, n-1} & S O_{0}(n+2) / S O(n) \times S O(2) & 2 & n-2 \\ & \operatorname{Herm}(3, \mathbb{O}) & E_{6} \cdot T / F_{4} & 3 & 8\end{array}$

Table 3

Simple reduced PJTS (not of Euclidean type)

\begin{tabular}{|c|c|c|c|c|c|}
\hline & $V$ & $X$ & $r$ & $a$ & $b$ \\
\hline $2 \leq s$ & $\mathbb{R}^{1 \times s}$ & $\mathbb{P}_{s}(\mathbb{R})$ & 1 & 0 & $s-1$ \\
\hline $2 \leq r \leq s$ & $\mathbb{R}^{r \times s}$ & $G r\left(r, \mathbb{R}^{r+s}\right)$ & $r$ & 2 & $s-r$ \\
\hline $2 \leq r$ & $\operatorname{Asym}(2 r, \mathbb{R})$ & $S O(2 r)$ & $r$ & 2 & 0 \\
\hline $2 \leq r$ & $\operatorname{Asym}(2 r+1, \mathbb{R})$ & $S O(2 r+1)$ & $r$ & 2 & 2 \\
\hline $2 \leq p \leq q$ & $\mathbb{R}^{p, q}$ & $Q^{p, q}(\mathbb{R})$ & $r$ & $\left\{\begin{array}{l}a_{+}=q-1 \\
a_{-}=p-1\end{array}\right.$ & 0 \\
\hline & $\mathbb{O}_{s}^{1 \times 2}$ & $G r\left(2, \mathbb{H}^{4}\right) / \mathbb{Z}_{2}$ & 2 & 6 & 4 \\
\hline & $\operatorname{Herm}\left(3, \mathbb{O}_{s}\right)$ & $S U(8) / S p(4) \cdot \mathbb{Z}_{2}$ & 3 & 4 & 1 \\
\hline
\end{tabular}


Table 4

\section{Simple non-reduced PJTS}

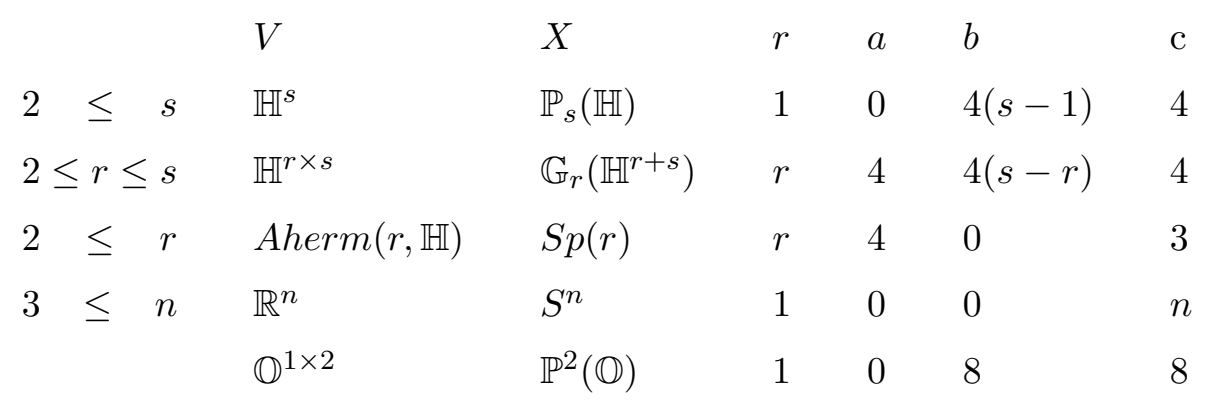

The classification of PJTS can be found in [6] Part II Table 4, or in [18]. See also [22], [23].

\section{References}

[1] Andrews G., Askey R. and Roy R., Special functions, Cambridge University Press (2000).

[2] Bertram, W., The geometry of Jordan and Lie structures, Lecture Notes in Mathematics, v. 1754 (2000), Springer Verlag

[3] Bopp N. and Rubenthaler H., Local zeta functions attached to the minimal spherical series for a class of symmetric spaces, Mem. Amer. Math. Soc. 174 (2005)

[4] De Oliveira Da Costa F., Quelques aspects géométriques et analytiques des domaines bornés symétriques, Thèse de l'Université Poincaré (Nancy) (2011)

[5] Faraut J. and Korányi A., Analysis on symmetric cones, Oxford mathematical monographs, Clarendon Press (1994)

[6] Faraut J., Kaneyuki S., Korányi A., Lu Qi-keng and Roos G., Analysis and geometry on complex homogeneous domains, Progress in Mathematics, Birkhäuser (2000)

[7] Faraut J. and Pevzner M. Berezin kernels and analysis on Makarevich spaces, Indag. Math. 16 (2005), 461-486 
[8] Forrester P. and Warnaar O., The importance of the Selberg integral, Bull. Amer. Math. Soc. 45 (2008) 489-534

[9] Helgason S. Groups and geometric analysis, Academic Press, New York, London (1984)

[10] Helwig K-H., Halbeinfache reelle Jordan-Algebren, Math. Z. 109 (1969), $1-28$

[11] Helwig K-H., Jordan-Algebren und symmetrische Räume I, Math. Z. 115 (1970), 315-349

[12] Johnson K.D. Degenerate principal series on compact groups, Math. Ann. 302 (1990), 703-718

[13] Johnson K.D. Composition series and intertwining operators for the spherical principal series. I, II, Trans. Amer. Math. Soc. 215 (1976), 269-283, 229 (1977), 137-173

[14] Knapp, A., Representation theory of semisimple groups, Princeton University Press (1986)

[15] Koecher M., An elementary approach to bounded symmetric domains Lecture Notes, Rice University (1969), Houston, Texas

[16] Loos O., Jordan triple systems, R-spaces and bounded symmetric domains, Bull. Amer. Math. Soc. 77 (1971), 558-561

[17] Loos O., Jordan Pairs, Lecture Notes in Mathematics, vol. 460, Springer Verlag (1975)

[18] Loos O., Bounded symmetric domains and Jordan pairs, Mathematical Lectures, University of California at Irvine (1977)

[19] Loos O., Charakterisierung symmetrischer R-Räume durch ihre Einheitsgitter, Math. Z. 189 (1985), 211-226

[20] Möllers J. and Schwarz B., Structure of the degenerate principal series on symmetric $R$-spaces, to appear

[21] Nagano T., Transformation groups on compact symmetric spaces, Trans. Amer. Math. Soc. 118 (1965), 428-453

[22] Neher N., Klassifikation der einfachen reellen speziellen JordanTripelsysteme, Manuscripta Math. 31 (1980), 197-215 
[23] Neher N., Klassifikation der einfachen reellen speziellen AusnahmeJordan-Tripelsysteme, J. Reine Angew. Math. 322 (1981), 145-169

[24] Ólafsson G. and Pasquale A., The $\operatorname{Cos}^{\lambda}$ and $\operatorname{Sin}^{\lambda}$ transforms as intertwining operators between the generalized principal series representations of $S L(N+1, \mathbb{K})$, to appear $(2012)$

[25] Ólafsson G., Pasquale A. and Rubin B., Analytic aspects of the Cosine transform, to appear (2012)

[26] Ørsted B. and Zhang G.K., Generalized principal series representations and tube domains, Duke Math. J. 78 (1995), 335-357

[27] Sahi S., Jordan algebras and degenerate principal series, J. Reine Angew. Math. 462 (1995), 1-18

[28] Satake I., Algebraic structures of Symmetric Domains, Iwanami Shoten, Tokyo and Princeton University Press, Princeton, NJ (1980)

[29] Takeuchi M., Cell decompositions and Morse equalities on certain symmetric spaces, J. Fac. Sci. Univ. Tokyo 12 (1965), 81-192

[30] Vogan D. and Wallach N., Intertwining operators for real reductive groups, Adv. in Math. 82 (1990), 203-243

[31] Wallach N., Real reductive groups II, Pure and Applied Mathematics, Academic Press, Boston (1992)

[32] Zhang G.K., Jordan algebras and generalized principal series representations, Math. Ann. 302 (1995), 773-786

[33] Zhang G.K. Radon, cosine and sine transforms on Grassmannian manifolds, to appear

Address

Jean-Louis Clerc

Institut Elie Cartan, Université de Lorraine, 54506 Vandœuvre-lès-Nancy, France

jean-louis.clerc@univ-lorraine.fr 